\title{
WAVE TRANSMISSION THROUGH RANDOM LAYERING WITH PRESSURE RELEASE BOUNDARY CONDITIONS
}

\author{
JOSSELIN GARNIER* AND KNUT SøLNA ${ }^{\dagger}$
}

\begin{abstract}
This paper considers the statistical properties of the waves generated by a point source in the subsurface and transmitted towards the surface through a randomly layered medium. The problem is analyzed in a regime of separation of scales and with pressure release boundary conditions at the surface. Using a probabilistic representation of the spectral density of the waves received at the surface, the transmitted intensity is analyzed. Conserved quantities specific to the pressure release boundary conditions are evaluated and the power delay spread is computed. In particular a waveguiding effect that is produced by the reflections from the surface and from the random medium is identified and analyzed. This effect is different from the propagation in a standard waveguide from the point of view of the decay of the intensity.
\end{abstract}

Key words. Wave propagation, random media, stochastic equations.

AMS subject classifications. 35R60, 86A15.

1. Introduction. Wave propagation in randomly layered media has been extensively studied because it is both a mathematically tractable problem and a physically relevant model, in particular for naturally occuring media such as the earth's crust, the atmosphere and the ocean. A separation of scales technique enables the asymptotic analysis of the statistical properties of the waves generated by a point source and transmitted through or reflected by a random medium $[1,4,6,7,8,9,13]$. In most papers regarding randomly layered media the boundary conditions are simple and the authors typically choose to use matched boundary conditions in which the boundaries are transparent. In this paper we show that the boundary conditions play a primary role in the wave transmission through a random medium and may change the picture quantitatively and qualitatively. Indeed, a partially or totally reflecting boundary condition in addition to the localization phenomenon can induce a waveguiding effect close to the surface $[11,15]$. Here, we will explain how the effects of the pressure release boundary conditions interact in an interesting way with the wave localization phenomenon in a randomly layered medium.

In geophysics a randomly layered medium is often used as a model for the earth's crust $[2,3,17,18]$. The boundary conditions at the surface are imposed by the large contrast in density between the earth and the atmosphere, which means that the pressure is vanishing and reflecting boundary conditions for the pressure field can be used [5]. This situation is briefly mentioned in [1] in the case of a source at the surface whose signal is reflected by the random medium. In our paper we address the transmission of the waves generated by a point source located in the subsurface. As we will see the problem can be analyzed using the separation of scales technique presented in [7] and leads to original results in terms of the transmitted intensity and the power delay spread (which is defined as the duration of the coda waves following the arrival of the direct waves).

The paper is organized as follows. In Section 2 we describe the acoustic model with the point source. We consider the wave transmitted through a random medium

${ }^{*}$ Laboratoire de Probabilités et Modèles Aléatoires \& Laboratoire Jacques-Louis Lions, Université Paris VII, 2 Place Jussieu, 75251 Paris Cedex 5, France garnier@math.jussieu.fr

${ }^{\dagger}$ Mathematics Department, University of California at Irvine, Irvine, CA 92697 ksolna@math.uci.edu 
in Section 3, where we give the integral representation for the vertical velocity field at the surface. In Section 4 we introduce some quantities of interest that we use to characterize the field at the surface. In Section 5 we present the key theoretical tool that allows us to analyze waves in random media, the spectral representation. In Section 6 we discuss some special cases that illustrate how the spectral density and its jump process interpretation relates to physical quantities such as the transmitted intensity. We give in Section 7 the main results of the paper that characterize some of the salient aspects of the interaction between the wave localization phenomenon and the pressure release surface boundary conditions. In particular we discuss the "trapping" of the wave by the pressure release boundary conditions and the medium heterogeneities. We finally compare the theoretical predictions of our asymptotic theory with numerical simulations in Section 8.

2. Acoustic Model. We consider linear acoustic waves propagating in a threedimensional layered medium and generated by a point source. The governing equations are

$$
\rho^{\varepsilon}(z) \frac{\partial \overrightarrow{\boldsymbol{u}}^{\varepsilon}}{\partial t}+\nabla p^{\varepsilon}+\sigma^{\varepsilon}(z) \overrightarrow{\boldsymbol{u}}^{\varepsilon}=\overrightarrow{\boldsymbol{F}}^{\varepsilon}(t, \boldsymbol{x}, z), \quad \frac{1}{K^{\varepsilon}(z)} \frac{\partial p^{\varepsilon}}{\partial t}+\nabla \cdot \overrightarrow{\boldsymbol{u}}^{\varepsilon}=0,
$$

where $p^{\varepsilon}$ is the pressure field, $\overrightarrow{\boldsymbol{u}}^{\varepsilon}$ is the three-dimensional velocity field, $\rho^{\varepsilon}$ is the density of the medium, $K^{\varepsilon}$ is the bulk modulus of the medium, $\sigma^{\varepsilon}$ is the dissipation, and $(\boldsymbol{x}, z) \in \mathbb{R}^{2} \times \mathbb{R}$ are the space coordinates. The parameters of the medium $\rho^{\varepsilon}$, $K^{\varepsilon}$, and $\sigma^{\varepsilon}$ vary only along the $z$-direction. The forcing term $\overrightarrow{\boldsymbol{F}}^{\varepsilon}$ models the source located in the subsurface $z<0$.

We consider in this paper the situation in which a layered medium occupying the section $z \in(-L, 0)$ is sandwiched in between two homogeneous half-spaces. The homogeneous half-space $z \leq-L$ is matched to the layered section $z \in(-L, 0)$. Motivated by geophysical applications we assume that the density $\rho_{a}$ in the homogeneous half-space $z \geq 0$ is much smaller than the density $\rho^{\varepsilon}(z)$ in the layered medium for $z \leq 0$. Since the velocity and pressure are continuous away from the sources, the pressure in $z>0$ goes to zero and hence, by continuity, also at $z=0$. These are the so-called pressure release boundary conditions.

We consider the case with a randomly layered medium in the region $z \in(-L, 0)$. The parameters of the medium are assumed to be of the form

$$
\begin{aligned}
\left(K^{\varepsilon}\right)^{-1}(z) & =\left\{\begin{array}{ll}
K^{-1}(z) & \text { if } z \in(-\infty,-L] \\
K^{-1}(z)\left[1+\nu\left(z, \frac{z}{\varepsilon^{2}}\right)\right] & \text { if } z \in(-L, 0)
\end{array},\right. \\
\rho^{\varepsilon}(z) & =\rho(z) \quad \text { if } z \in(-\infty, 0), \\
\sigma^{\varepsilon}(z) & =\sigma(z) \quad \text { if } z \in(-\infty, 0) .
\end{aligned}
$$

In this model the parameters of the medium have two types of variations: on the one hand, deterministic, slow and smooth variations with a typical scale of variation of the same order as the thickness $L$ of the layer, and on the other hand random and rapid fluctuations with a typical scale of variation much smaller than the thickness of the layer. The small dimensionless parameter $\varepsilon^{2}$ is the ratio between these two scales. The small-scale random fluctuations are described by the random process $\nu\left(z, z^{\prime}\right)$. The process $\nu$ is bounded in magnitude by a constant less than one, so that $K^{\varepsilon}$ is a positive quantity. For each $z$, the random process $z^{\prime} \mapsto \nu\left(z, z^{\prime}\right)$ is stationary and zero mean and it has strong mixing properties. The $z$-dependence of $\nu\left(z, z^{\prime}\right)$ models a depth-dependence of the statistical properties of the medium. The important quantity 
from the statistical point of view is the integrated covariance of the fluctuations of the random medium defined by

$$
\gamma(z)=\int_{-\infty}^{\infty} \mathbb{E}\left[\nu(z, 0) \nu\left(z, z^{\prime}\right)\right] d z^{\prime} .
$$

By the Wiener-Khintchine theorem it is nonnegative valued. The integrated covariance $\gamma(z)$ plays the role of a depth-dependent scattering coefficient. As will become clear in the sequel, the statistics of the wave field depends on the random medium via this integrated covariance.

The source is modeled by the forcing term $\overrightarrow{\boldsymbol{F}}^{\varepsilon}$. We assume that it is point-like, located at $(\boldsymbol{x}, z)=(\mathbf{0},-L)$, and that it emits a short pulse whose carrier wavelength is of order $\varepsilon$ :

$$
\overrightarrow{\boldsymbol{F}}^{\varepsilon}(t, \boldsymbol{x}, z)=\overrightarrow{\boldsymbol{f}}^{\varepsilon}(t) \delta(z+L) \delta(\boldsymbol{x}), \quad \overrightarrow{\boldsymbol{f}}^{\varepsilon}(t)=\varepsilon^{1 / 2} \overrightarrow{\boldsymbol{f}}\left(\frac{t}{\varepsilon}\right),
$$

where we assume that the support of the Fourier transform of $\overrightarrow{\boldsymbol{f}}=\left(\boldsymbol{f}_{\boldsymbol{x}}, f_{z}\right)$ is bounded away from zero and of rapid decay at infinity. The particular scaling of $\overrightarrow{\boldsymbol{f}}^{\varepsilon}$ in (2.6) means that the carrier wavelength is large compared to the microscopic scale of variation of the random fluctuations of the medium and small compared to the macroscopic scale of variation of the background medium. The normalizing amplitude factor $\varepsilon^{1 / 2}$ multiplying the source term makes the total energy $\int\left|\partial_{t} \overrightarrow{\boldsymbol{f}}^{\varepsilon}(t)\right|^{2} d t$ released by the point source independent of $\varepsilon$.

We observe the vertical velocity field at the surface $z=0$ :

$$
u^{\varepsilon}(t, \boldsymbol{x})=\overrightarrow{\boldsymbol{e}}_{z} \cdot \overrightarrow{\boldsymbol{u}}^{\varepsilon}(t, \boldsymbol{x}, z=0),
$$

where $\overrightarrow{\boldsymbol{e}}_{z}$ is the unit vector $(\mathbf{0}, 1)$ that is the outward unit normal vector of the surface. This is typical of seismographs, which usually record only the vertical velocity because it is less noisy and gives better records of some seismic waves. Note, moreover, that with the idealized pressure release boundary conditions the horizontal velocity components vanish along with the pressure at the surface.

3. Propagation though a Dissipative Layered Medium. We define the background velocity and impedance by

$$
c(z)=\frac{\sqrt{K(z)}}{\sqrt{\rho(z)}}, \quad \zeta(z)=\sqrt{K(z) \rho(z)} .
$$

The background bulk modulus $K(z)$ and density $\rho(z)$ in (2.2-2.4) are smooth. This implies that the reflections induced by the macroscopic variations of $K^{\varepsilon}, \rho^{\varepsilon}$, and $\sigma^{\varepsilon}$ are extremely small, they are dominated by incoherent reflections that are generated by the interaction of the waves with the microscopic random fluctuations of the medium modeled by the random process $\nu$.

3.1. Integral Representation of the Recorded Field. The vertical velocity field recorded at the surface $z=0$ can be shown (see Appendix A) to have the integral representation

$$
\begin{aligned}
u^{\varepsilon}(t, \boldsymbol{x}) & =\frac{1}{(2 \pi)^{3} \varepsilon^{3 / 2}} \iint \hat{g}^{\varepsilon}(\omega, \boldsymbol{\kappa}) \mathcal{T}_{\omega, \kappa}^{\varepsilon} \exp \left(-i \frac{\omega}{\varepsilon}(t-\boldsymbol{\kappa} \cdot \boldsymbol{x})\right) \omega^{2} d \boldsymbol{\kappa} d \omega, \\
\hat{g}^{\varepsilon}(\omega, \boldsymbol{\kappa}) & =\frac{\hat{f}_{z}(\omega)}{\zeta^{1 / 2}(\kappa,-L) \zeta^{1 / 2}(\kappa, 0)}+\frac{i \omega \boldsymbol{\kappa} \cdot \hat{\boldsymbol{f}}_{\boldsymbol{x}}(\omega)}{i \omega \rho(-L)-\varepsilon \sigma(-L)} \frac{\zeta^{1 / 2}(\kappa,-L)}{\zeta^{1 / 2}(\kappa, 0)}
\end{aligned}
$$


Here, we have used the notation $\kappa=|\boldsymbol{\kappa}|$, and:

- The Fourier transforms are defined by

$$
\hat{f}_{z}(\omega)=\int f_{z}(t) e^{i \omega t} d t, \quad \hat{\boldsymbol{f}}_{\boldsymbol{x}}(\omega)=\int \boldsymbol{f}_{\boldsymbol{x}}(t) e^{i \omega t} d t .
$$

- $\zeta(\kappa, z)$ and $c(\kappa, z)$ are the mode- and depth-dependent impedance and velocity

$$
\zeta(\kappa, z)=\frac{\zeta(z)}{\sqrt{1-\kappa^{2} c(z)^{2}}}, \quad c(\kappa, z)=\frac{c(z)}{\sqrt{1-\kappa^{2} c(z)^{2}}} .
$$

- $\tau(\kappa, z)$ is the mode-dependent vertical travel time from the surface to the depth $z$ :

$$
\tau(\kappa, z)=\int_{0}^{z} \frac{d z^{\prime}}{c\left(\kappa, z^{\prime}\right)},
$$

which is negative for $z<0$.

- The random complex coefficient $\mathcal{T}_{\omega, \kappa}^{\varepsilon}$ is the generalized transmission coefficient (for pressure release boundary conditions). It captures the interaction of the wave with the medium, both the scattering, the damping, and the pressure release boundary conditions. It can be expressed in terms of the mode-dependent reflection and transmission coefficients $R_{\omega, \kappa}^{\varepsilon}$ and $T_{\omega, \kappa}^{\varepsilon}$ of the random section (for matched boundary conditions, that is, transparent boundary conditions), which are described in detail in Appendix A, in the following way:

$$
\mathcal{T}_{\omega, \kappa}^{\varepsilon}=\exp \left(-i \frac{\omega}{\varepsilon} \tau(\kappa,-L)\right) \frac{T_{\omega, \kappa}^{\varepsilon}}{1-R_{\omega, \kappa}^{\varepsilon}} .
$$

We describe the statistial properties of the generalized transmission coefficient $\mathcal{T}_{\omega, \kappa}^{\varepsilon}$ in Section 5. We remark that the expansion

$$
\mathcal{T}_{\omega, \kappa}^{\varepsilon}=\sum_{j=0}^{\infty} \exp \left(-i \frac{\omega}{\varepsilon} \tau(\kappa,-L)\right) T_{\omega, \kappa}^{\varepsilon}\left(R_{\omega, \kappa}^{\varepsilon}\right)^{j}
$$

reflects the physical situation that the waves recorded at the surface are the sum of the contributions of the waves that have been transmitted through the random medium from the source plane $z=-L$ to the surface $z=0$ (term $j=0$ ) and the contributions of the waves that have reached the surface and have then been reflected back and forth by the surface and by the random region generating "multiples" (see Figure 3.1). These reflections and multiples arise due to the pressure release boundary conditions at the surface which is an important ingredient in our model.

- The integral in $\boldsymbol{\kappa}$ in (3.2) is limited to $\kappa<\kappa_{\max }$ for

$$
\kappa_{\max }=\min _{z \in(-L, 0)} c^{-1}(z) .
$$

The restriction $\kappa<c^{-1}(-L)$ comes from the fact that modes generated with transverse slownesses that violate this condition are exponentially damped and can be neglected. The restriction $\kappa<\kappa_{\max }$ means that there are no turning points in the medium for the mode with slowness $\kappa$. Modes generated with transverse slownesses that violate this condition do not reach the surface, so they can be neglected (remember that the random medium is layered so that scattering does not change the slowness). 


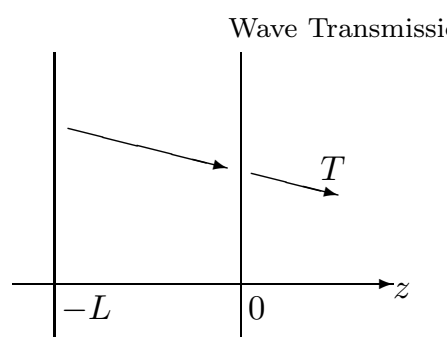

Matched boundary conditions

FIG. 3.1. Schematic of the geometric configuration: The source is in the plane $z=-L$, the medium is random in the section $z \in(-L, 0)$, and the interface $z=0$ is transparent (left, matched boundary conditions) or reflecting (right, pressure release boundary conditions).

3.2. Special Case with Dissipative Homogeneous Medium. In this section we consider the particular case with the density, bulk modulus, and dissipation of the medium being constant and denoted by $\rho_{0}, K_{0}$, and $\sigma_{0}$ for $z<0$, moreover, with pressure release boundary conditions. The vertical velocity field recorded at the surface $z=0$ then has the integral representation (see Appendix A):

$$
\begin{array}{r}
u^{\varepsilon}(t, \boldsymbol{x})=\frac{1}{(2 \pi)^{3} \varepsilon^{3 / 2}} \iint\left[\frac{\hat{f}_{z}(\omega)}{\zeta_{0}(\kappa)}+\frac{i \omega}{i \omega \rho_{0}-\varepsilon \sigma_{0}} \boldsymbol{\kappa} \cdot \hat{\boldsymbol{f}}_{\boldsymbol{x}}(\omega)\right] \exp \left(-i \frac{\omega}{\varepsilon}(t-\boldsymbol{\kappa} \cdot \boldsymbol{x})\right) \\
\quad \times \exp \left(i \frac{\omega}{\varepsilon} \frac{L}{c_{0}(\kappa)}-\frac{\sigma_{0} c_{0}(\kappa)}{2 \zeta_{0} c_{0}} L\right) \omega^{2} d \boldsymbol{\kappa} d \omega .
\end{array}
$$

In this expression:

- The constant background parameters are

$$
c_{0}=\frac{\sqrt{K_{0}}}{\sqrt{\rho_{0}}}, \quad c_{0}(\kappa)=\frac{c_{0}}{\sqrt{1-c_{0}^{2} \kappa^{2}}}, \quad \zeta_{0}=\sqrt{K_{0} \rho_{0}}, \quad \zeta_{0}(\kappa)=\frac{\zeta_{0}}{\sqrt{1-c_{0}^{2} \kappa^{2}}} .
$$

- $L / c_{0}(\kappa)$ is the mode-dependent travel time from the source to the surface.

- $\exp \left[-\sigma_{0} c_{0}(\kappa) /\left(2 \zeta_{0} c_{0} L\right)\right]$ is the damping of the waves propagating from the sources to the surface.

Recall that we consider the limit $\varepsilon \rightarrow 0$. In absence of microscopic random fluctuations of the medium this is simply a high-frequency regime. We now exploit this to obtain a simplified expression for the transmitted field by applying the stationary phase method. We find that the leading order value for the integral (3.8) is given by the contribution around the stationary slowness vector

$$
\boldsymbol{\kappa}_{\mathrm{sp}}=\frac{1}{c_{0}} \frac{\boldsymbol{x}}{\sqrt{L^{2}+|\boldsymbol{x}|^{2}}},
$$

and this gives a contribution that is concentrated around the critical time

$$
t_{\mathrm{sp}}=\frac{\sqrt{L^{2}+|\boldsymbol{x}|^{2}}}{c_{0}}
$$

that is, the travel time from the source to the observation point. Denoting the source point, $(\mathbf{0},-L)$, by $S$ and the point of observation, $(\boldsymbol{x}, 0)$, by $M$ we get that the wave energy is received only around the travel time $S M / c_{0}$ in the form of a short pulse:

$$
\lim _{\varepsilon \rightarrow 0} \varepsilon^{1 / 2} u^{\varepsilon}\left(\frac{S M}{c_{0}}+\varepsilon s, \boldsymbol{x}\right)=\frac{L}{2 \pi \zeta_{0} c_{0} S M^{2}} e^{-\frac{\sigma_{0}}{2 \zeta_{0}} S M} \frac{\overrightarrow{S M} \cdot \overrightarrow{\boldsymbol{f}^{\prime}}(s)}{S M},
$$


where $\overrightarrow{S M}=(\boldsymbol{x}, L), S M=\sqrt{L^{2}+|\boldsymbol{x}|^{2}}$, and the prime stands here for the timederivative. This is characteristic of wave propagation in homogeneous media in the high-frequency regime. From this follows the expression of the high-frequency Green's function in the homogeneous medium with pressure release boundary conditions. With matched boundary conditions (MBC), which corresponds to a transparent interface instead of a reflecting interface for the pressure, we find the same expression up to a factor $1 / 2$ :

$$
\left.\lim _{\varepsilon \rightarrow 0} \varepsilon^{1 / 2} u^{\varepsilon}\left(\frac{S M}{c_{0}}+\varepsilon s, \boldsymbol{x}\right)\right|_{\mathrm{MBC}}=\frac{L}{4 \pi \zeta_{0} c_{0} S M^{2}} e^{-\frac{\sigma_{0}}{2 \varepsilon_{0}} S M} \frac{\overrightarrow{S M} \cdot \overrightarrow{\boldsymbol{f}}^{\prime}(s)}{S M} .
$$

This factor $1 / 2$ comes from the fact that the vertical velocity field for the pressure release boundary conditions is the superposition of the right-going wave that comes from the source and of the left-going wave that is reflected by the surface.

A more detailed discussion of the stationary phase calculation, the lateral slowness and related concepts can be found in [7, Chapter $14 \& 17]$.

4. Some Quantities of Interest. In this section we introduce some quantities that subsequently will be used to characterize the effects of the free boundary and the wave scattering by the random medium.

The transmitted wave statistics is characterized by the statistical distribution of the generalized transmission coefficient. In the asymptotic regime $\varepsilon \rightarrow 0$ the generalized spectral density $\mathcal{U}$ (for pressure release boundary conditions) associated with the generalized transmission coefficient plays a central role and it is defined by

$$
\mathbb{E}\left[\mathcal{T}_{\omega, \kappa+\varepsilon \lambda / 2}^{\varepsilon} \overline{\mathcal{T}_{\omega, \kappa-\varepsilon \lambda / 2}^{\varepsilon}}\right] \stackrel{\varepsilon \rightarrow 0}{\longrightarrow} \int \mathcal{U}(\omega, \kappa, \xi) e^{-i \omega \kappa \lambda \xi} d \xi,
$$

where the convergence holds in the sense of distribution with respect to frequency $\omega$ and slowness $\kappa$. We describe the spectral density and its relation to an underlying Markov jump stochastic process in detail in Section 5. We remark that, as explained below, our focus will be on wave intensities and energies for which the spectral density in (4.1) based on an offset in slowness only, and not in frequency, is the appropriate one [7, Chapter 14]. Had we focused on the local power spectrum in general we would have had to use an offset in frequency also, as in $[1,7]$.

The square modulus of the generalized transmission coefficient, $\left|\mathcal{T}_{\omega, \kappa}^{\varepsilon}\right|^{2}$, the power transmission coefficient, is an important physical quantity that describes how wave power, at a particular frequency and lateral slowness, is transmitted from the source to the surface. Using the probabilistic representation of the spectral density in terms of a jump process we discuss the statistical properties of the power transmission coefficient in Subsection 7.1. We show in particular that the mean power transmission coefficient is equal to one whatever the scattering properties of the medium in the absence of dissipation.

In Section 6 we study the mean intensity observed at the surface, also in the asymptotic regime $\varepsilon \rightarrow 0$ :

$$
I(t, \boldsymbol{x})=\lim _{\varepsilon \rightarrow 0} \mathbb{E}\left[u^{\varepsilon}(t, \boldsymbol{x})^{2}\right]
$$

We remark that

1) The convergence holds in the sense of distribution with respect to time $t$ and position $\boldsymbol{x}$, and the mean time-dependent intensity $t \rightarrow I(t, \boldsymbol{x})$ possesses a singular 
component concentrated at the background travel time $\tau(S, M)$ from the source point $S=(-L, \mathbf{0})$ to the observation point $M=(0, \boldsymbol{x})$ and an absolutely continuous component supported in the semi-infinite interval starting from the background travel time $\tau(S, M)$. The singular component corresponds to the contributions of the direct waves and the absolutely continuous component correspond to the contributions of the coda waves.

2) The intensity $u^{\varepsilon}(t, \boldsymbol{x})^{2}$ is not a statistically stable quantity. If we apply the same method as in Section 9.3.4 [7], then one finds that the intensity $u^{\varepsilon}(t, \boldsymbol{x})^{2}$ at a time $t>\tau(S, M)$ obeys an exponential distribution in the limit $\varepsilon \rightarrow 0$.

3 ) We have in the case when the medium in the region $(-L, 0)$ has constant background velocity and impedance $c_{0}$ and $\zeta_{0}$, but possibly varying dissipation and scattering coefficients $\sigma(z)$ and $\gamma(z)$, that the mean intensity is given in terms of the spectral density $\mathcal{U}$ by

$$
I(t, \boldsymbol{x})=\frac{1}{(2 \pi)^{3} c_{0}^{2} t^{2}} \int \mathcal{U}\left(\omega, \frac{|\boldsymbol{x}|}{c_{0}^{2} t}, c_{0}^{2} t\right)\left|\frac{\hat{f}_{z}(\omega)}{\zeta_{0}\left(\frac{|\boldsymbol{x}|}{c_{0}^{2} t}\right)}+\frac{\boldsymbol{x}}{\zeta_{0} c_{0} t} \cdot \hat{\boldsymbol{f}}_{\boldsymbol{x}}(\omega)\right|^{2} \omega^{2} d \omega .
$$

This representation is derived in Appendix C.

It can be shown that the decoherence time and length of the intensity are short, of the order of $\varepsilon$, see (C.3). The short decoherence time and length in turn give the self-averaging property, or statistical stability, of averaged quantities. In Appendix D we prove the self-averaging property of time-space averaged quantities: If $\phi(\boldsymbol{x})$ and $\psi(t)$ are two smooth compactly supported test functions, then

$$
\lim _{\varepsilon \rightarrow 0} \iint \phi(\boldsymbol{x}) \psi(t) u^{\varepsilon}(t, \boldsymbol{x})^{2} d \boldsymbol{x} d t \stackrel{\varepsilon \rightarrow 0}{\longrightarrow} \iint \phi(\boldsymbol{x}) \psi(t) I(t, \boldsymbol{x}) d \boldsymbol{x} d t, \quad \text { in probability. }
$$

This property is important because it indicates that space- and/or time-averaged quantities should be used for imaging or communication in order to minimize random fluctuations.

In Section 7.2 we discuss the total energy transmitted to the surface defined by

$$
\mathcal{E}^{\varepsilon}=\frac{\zeta(0)}{2} \iint u^{\varepsilon}(t, \boldsymbol{x})^{2} d \boldsymbol{x} d t
$$

We deduce the remarkable fact that this quantity does not depend on the details of the microscale medium fluctuations model in the non-dissipative case. We show moreover that this quantity is statistically stable so that it does not depend on the particular realization of the random medium. Thus, the transmitted energy is invariant with respect the microscale. This result is valid in the regime $\varepsilon \rightarrow 0$.

Then, in Section 7.3, we discuss the mean energy density associated with the vertical velocity field transmitted to the surface. We compensate the density for loss due to geometric spreading and define the "modified" surface energy density $\int t^{2} I(t, \boldsymbol{x}) d t$. The interesting fact is that this quantity also does not depend on the details of the model of the microscale medium fluctuations in the non-dissipative case, it coincides with the density in the case without microscale fluctuations.

5. Statistical Analysis of the Generalized Transmission Coefficient. We consider the model in (2.2-2.4) with a smooth background medium modulated by random medium fluctuations. The spectral representation of the wave constitutes the central theoretical tool to describe wave statistics and the derivation of the trapping 
results are based on this. Here, we present the basic theory associated with the spectral representation. This is an extension of results in [7] which incorporates the effect of the pressure release boundary conditions. We start by the following proposition.

Proposition 5.1. The autocorrelation function of the generalized transmission coefficient at two nearby slownesses satisfies

$$
\mathbb{E}\left[\mathcal{T}_{\omega, \kappa+\varepsilon \lambda / 2}^{\varepsilon} \overline{\mathcal{T}_{\omega, \kappa-\varepsilon \lambda / 2}^{\varepsilon}}\right] \stackrel{\varepsilon \rightarrow 0}{\longrightarrow} \int \mathcal{U}(\omega, \kappa, \xi) e^{-i \omega \kappa \lambda \xi} d \xi
$$

The spectral density $\mathcal{U}(\omega, \kappa, \xi)$ has the probabilistic representation:

$$
\begin{aligned}
\mathcal{U}(\omega, \kappa, \xi)= & \mathbb{E}\left[\delta\left(\xi-\int_{-L}^{0} c(\kappa, z)\left(2 N_{\omega, \kappa}(z)+1\right) d z\right)\right. \\
& \left.\times \exp \left(-\int_{-L}^{0} \frac{\sigma(z) c(\kappa, z)}{\zeta(z) c(z)}\left(2 N_{\omega, \kappa}(z)+1\right) d z\right) \mid N_{\omega, \kappa}(-L)=0\right]
\end{aligned}
$$

where $\left(N_{\omega, \kappa}(z)\right)_{-L \leq z \leq 0}$ is the jump Markov process with state space $\mathbb{N}$ and inhomogeneous infinitesimal generator

$$
\mathcal{L}_{z} \phi(N)=\frac{\gamma(z) c(\kappa, z)^{2} \omega^{2}}{4 c(z)^{4}}\left[(N+1)^{2}(\phi(N+1)-\phi(N))+N^{2}(\phi(N-1)-\phi(N))\right]
$$

The proof of Proposition 5.1 is given in Appendix B. From the probabilistic representation (5.2) we can also write using the Kolmogorov equation that $\mathcal{U}(\omega, \kappa, \xi)=$ $\mathcal{U}_{0}(\omega, \kappa, \xi, 0)$, where $\mathcal{U}_{j}, j \geq 0$, are the solutions of the system of transport equations

$$
\begin{gathered}
\frac{\partial \mathcal{U}_{j}}{\partial z}+(2 j+1) c(\kappa,-L-z) \frac{\partial \mathcal{U}_{j}}{\partial \xi}=-\frac{(2 j+1) \sigma(-L-z)}{\zeta(-L-z)} \frac{c(\kappa,-L-z)}{c(-L-z)} \mathcal{U}_{j} \\
+\frac{\gamma(-L-z) c(\kappa,-L-z)^{2} \omega^{2}}{4 c(-L-z)^{4}}\left((j+1)^{2}\left(\mathcal{U}_{j+1}-\mathcal{U}_{j}\right)+j^{2}\left(\mathcal{U}_{j-1}-\mathcal{U}_{j}\right)\right)
\end{gathered}
$$

starting from $\mathcal{U}_{j}(\omega, \kappa, \xi, z=-L)=\delta(\xi)$ for all $j \in \mathbb{N}$.

The integral in $\xi$ of the spectral density $\mathcal{U}$ gives the expectation of the square modulus of the generalized transmission coefficient in the limit $\varepsilon \rightarrow 0$ :

$$
\begin{aligned}
\lim _{\varepsilon \rightarrow 0} \mathbb{E}\left[\left|\mathcal{T}_{\omega, \kappa}^{\varepsilon}\right|^{2}\right] & =\int \mathcal{U}(\omega, \kappa, \xi) d \xi \\
& =\mathbb{E}\left[\exp \left(-\int_{-L}^{0} \frac{\sigma(z) c(\kappa, z)}{\zeta(z) c(z)}\left(2 N_{\omega, \kappa}(z)+1\right) d z\right) \mid N_{\omega, \kappa}(-L)=0\right]
\end{aligned}
$$

The function $\xi \rightarrow \mathcal{U}(\omega, \kappa, \xi)$ is a scaled Fourier transform of the autocorrelation function of the generalized transmission coefficient at two nearby slownesses. It is a nonnegative valued distribution. In the non-dissipative case it can be interpreted as the probability density function of a random variable defined as a functional of the jump Markov process $N_{\omega, \kappa}$. This interpretation gives both theoretical and numerical tools to study the spectral density. The system of transport equations (5.4) is the Kolmogorov equation associated with this jump process. It does not describe a physical transport of energy directly, but it is a way to compute the spectral density (another way is to carry out Monte Carlo simulations of the jump process). 
In general, for a fixed pair $(\omega, \kappa)$, the function $\xi \rightarrow \mathcal{U}(\omega, \kappa, \xi)$ is a distribution with a singular component and an absolutely continuous component:

LEMMA 5.2. We have

$$
\mathcal{U}(\omega, \kappa, \xi)=\mathcal{U}_{s}(\omega, \kappa, \xi)+\mathcal{U}_{c}(\omega, \kappa, \xi)
$$

with

$$
\begin{array}{r}
\mathcal{U}_{s}(\omega, \kappa, \xi)=\delta\left(\xi-\int_{-L}^{0} c(\kappa, z) d z\right) \exp \left(-\int_{-L}^{0} \frac{\sigma(z) c(\kappa, z)}{\zeta(z) c(z)} d z\right) \\
\times \exp \left(-\int_{-L}^{0} \frac{\gamma(z) c(\kappa, z)^{2} \omega^{2}}{4 c(z)^{4}} d z\right)
\end{array}
$$

and

$$
\begin{aligned}
\mathcal{U}_{c}(\omega, \kappa, \xi)= & \exp \left(-\int_{-L}^{0} \frac{\sigma(z) c(\kappa, z)}{\zeta(z) c(z)} d z\right) \\
\times & \int_{-L}^{0} \frac{\gamma\left(z_{0}\right) c\left(\kappa, z_{0}\right)^{2} \omega^{2}}{4 c\left(z_{0}\right)^{4}} \exp \left(-\int_{-L}^{z_{0}} \frac{\gamma(z) c(\kappa, z)^{2} \omega^{2}}{4 c(z)^{4}} d z\right) \\
& \times \mathbb{E}\left[\delta\left(\xi-2 \int_{z_{0}}^{0} c(\kappa, z) N_{\omega, \kappa}(z) d z-\int_{-L}^{0} c(\kappa, z) d z\right)\right. \\
& \left.\quad \times \exp \left(-2 \int_{z_{0}}^{0} \frac{\sigma(z) c(\kappa, z)}{\zeta(z) c(z)} N_{\omega, \kappa}(z) d z\right) \mid N_{\omega, \kappa}\left(z_{0}\right)=1\right] d z_{0} .
\end{aligned}
$$

The singular component in (5.6) can be obtained from the probabilistic representation (5.2) as the contributions of the trajectories $\left(N_{\omega, \kappa}(z)\right)_{-L \leq z \leq 0}$ that do not jump. In the expression (4.2) of the mean transmitted intensity this is the contributions of the direct waves that constitute the wave front and that are received around the background travel time $\int_{-L}^{0} c(\kappa, z) d z$.

The absolutely continuous component in (5.7) can also be obtained from the probabilistic representation (5.2) as the contributions of the trajectories that jump at least once. In the expression (4.2) of the mean transmitted intensity this is the contributions of the waves that constitute the coda waves. Since $N_{\omega, \kappa}(z)$ takes nonnegative values, the support of the absolutely continuous component $\mathcal{U}_{c}$ is $\left[\int_{-L}^{0} c(\kappa, z) d z, \infty\right)$. In the case of a weakly scattering medium, i.e.

$$
\int_{-L}^{0} \frac{\gamma(z) c(\kappa, z)^{2} \omega^{2}}{4 c(z)^{4}} d z \ll 1
$$

we have

$$
\begin{aligned}
& \mathcal{U}_{c}(\omega, \kappa, \xi) \simeq \exp \left(-\int_{-L}^{0} \frac{\sigma(z) c(\kappa, z)}{\zeta(z) c(z)} d z\right) \int_{-L}^{0} \frac{\gamma\left(z_{0}\right) c\left(\kappa, z_{0}\right)^{2} \omega^{2}}{4 c\left(z_{0}\right)^{4}} \\
& \quad \times \delta\left(\xi-2 \int_{z_{0}}^{0} c(\kappa, z) d z-\int_{-L}^{0} c(\kappa, z) d z\right) \exp \left(-2 \int_{z_{0}}^{0} \frac{\sigma(z) c(\kappa, z)}{\zeta(z) c(z)} d z\right) d z_{0} .
\end{aligned}
$$

In the probabilistic interpretation this is the contribution of paths that jump exactly once. In the expression (4.2) of the mean transmitted intensity this corresponds to the first order reflections from the medium, that is, of the directly transmitted wave that is returned by the pressure release boundary conditions and finally returned to the surface again. 
6. Spectral Density and Mean Intensity. We illustrate in this section how the wave field components can be interpreted explicitly in terms of the spectral density and its jump process representation in the case of additional homogeneity assumptions.

6.1. Homogeneous Dissipative Medium. We here briefly revisit the case of a dissipative homogeneous medium, for which the spectral density is

$$
\mathcal{U}_{\mathrm{hom}}(\omega, \kappa, \xi)=\delta\left(\xi-c_{0}(\kappa) L\right) \exp \left(-\frac{\sigma_{0} c_{0}(\kappa)}{\zeta_{0} c_{0}} L\right)
$$

as shown by Proposition 5.1 (in the case $N_{\omega, \kappa}(z)=0$ ). Therefore

$$
\begin{aligned}
I_{\text {hom }}(t, \boldsymbol{x})= & \frac{L^{2}}{(2 \pi)^{3} c_{0}^{2} \zeta_{0}^{2}\left(L^{2}+|\boldsymbol{x}|^{2}\right)^{2}} \exp \left(-\frac{\sigma_{0}}{\zeta_{0}} \sqrt{L^{2}+|\boldsymbol{x}|^{2}}\right) \\
& \times \int\left|\frac{L \hat{f}_{z}(\omega)+\boldsymbol{x} \cdot \hat{\boldsymbol{f}}_{\boldsymbol{x}}(\omega)}{\sqrt{L^{2}+|\boldsymbol{x}|^{2}}}\right|^{2} \omega^{2} d \omega \delta\left(t-\frac{\sqrt{L^{2}+|\boldsymbol{x}|^{2}}}{c_{0}}\right) .
\end{aligned}
$$

This means that the signal emitted by the source at $S=(\mathbf{0},-L)$ is received at position $M=(\boldsymbol{x}, 0)$ at the time $\sqrt{L^{2}+|\boldsymbol{x}|^{2}} / c_{0}=S M / c_{0}$, which is the travel time between the two points $S$ and $M$. The intensity exhibits

- the power law decay $\left[\overrightarrow{S M} \cdot \overrightarrow{\boldsymbol{e}}_{z} / S M^{2}\right]^{2}$ due to geometric spreading,

- the exponential decay $\exp \left[-\left(\sigma_{0} / \zeta_{0}\right) S M\right]$ due to dissipation.

In absence of dissipation, if we compensate for the algebraic decay due to geometric spreading, then the "modified" surface energy density at $\boldsymbol{x}=\mathbf{0}$ is

$$
\int t^{2} I_{\mathrm{hom}}(t, \mathbf{0}) d t=\frac{1}{(2 \pi)^{3} c_{0}^{4} \zeta_{0}^{2}} \int\left|\hat{f}_{z}(\omega)\right|^{2} \omega^{2} d \omega .
$$

We will see in the next sections that the scattering induced by the random fluctuations in the medium does not modify the "modified" energy density received at the surface.

6.2. Mean Intensity in a Statistically Homogeneous Dissipative Random Medium. In this subsection we consider the case in which the medium in the region $z \in(-L, 0)$ is statistically homogeneous with constant scattering coefficient $\gamma_{0}$, background velocity $c_{0}$, impedance $\zeta_{0}$, and dissipation $\sigma_{0}$. We have the probabilistic representation for the spectral density $\mathcal{U}$ :

$$
\mathcal{U}(\omega, \kappa, \xi)=\mathbb{E}\left[\delta\left(\xi-c_{0}(\kappa) \int_{-L}^{0}\left(2 N_{\omega, \kappa}(z)+1\right) d z\right) \mid N_{\omega, \kappa}(-L)=0\right] \exp \left(-\frac{\sigma_{0}}{\zeta_{0} c_{0}} \xi\right)
$$

where $\left(N_{\omega, \kappa}(z)\right)_{-L \leq z \leq 0}$ is the jump Markov process with state space $\mathbb{N}$ and homogeneous infinitesimal generator:

$$
\mathcal{L}_{\omega, \kappa} \phi(N)=\frac{\gamma_{0} c_{0}(\kappa)^{2} \omega^{2}}{4 c_{0}^{4}}\left[(N+1)^{2}(\phi(N+1)-\phi(N))+N^{2}(\phi(N-1)-\phi(N))\right] .
$$

We note first that, since $N_{\omega, \kappa}(z)$ takes nonnegative values, the function $\xi \rightarrow$ $\mathcal{U}(\omega, \kappa, \xi)$ is supported in $\left[c_{0}(\kappa) L, \infty\right)$, and therefore the function $t \rightarrow I(t, \boldsymbol{x})$ is supported in $\left[\sqrt{L^{2}+|\boldsymbol{x}|^{2}} / c_{0}, \infty\right)$. This can be interpreted as a consequence of the hyperbolicity of the acoustic wave equations with background velocity $c_{0}$.

We also note that from the probabilistic representation (6.3) and the integral representation (4.2) the expression of the mean transmitted intensity $I(t, \boldsymbol{x})$ can be 
deduced from the one in the absence of dissipation by a simple multiplication by the damping factor $\exp \left(-\sigma_{0} c_{0} t / \zeta_{0}\right)$.

There are two contributions in the mean transmitted intensity:

$$
I(t, \boldsymbol{x})=I_{s}(t, \boldsymbol{x})+I_{c}(t, \boldsymbol{x}) .
$$

1) $I_{s}$ is the contribution of the direct waves. It is a singular contribution received at the travel time $t=\sqrt{L^{2}+|\boldsymbol{x}|^{2}} / c_{0}$ (it is singular because it is supported on a time window of the order of the pulse width which is of order $\varepsilon$ ). It corresponds to the contributions of the trajectories of the jump process that do not jump

$$
\begin{aligned}
I_{s}(t, \boldsymbol{x})= & \delta\left(t-\frac{\sqrt{L^{2}+|\boldsymbol{x}|^{2}}}{c_{0}}\right) \frac{L^{2}}{(2 \pi)^{3} \zeta_{0}^{2} c_{0}^{2}\left(L^{2}+|\boldsymbol{x}|^{2}\right)^{2}} \exp \left(-\frac{\sigma_{0}}{\zeta_{0}} \sqrt{L^{2}+|\boldsymbol{x}|^{2}}\right) \\
& \times \int\left|\frac{L \hat{f}_{z}(\omega)+\boldsymbol{x} \cdot \hat{\boldsymbol{f}}_{\boldsymbol{x}}(\omega)}{\sqrt{L^{2}+|\boldsymbol{x}|^{2}}}\right|^{2} \exp \left(-\frac{\gamma_{0} \omega^{2}}{4 c_{0}^{2}} \sqrt{L^{2}+|\boldsymbol{x}|^{2}}\right) \omega^{2} d \omega .
\end{aligned}
$$

For a source pulse with small bandwidth $B$ and carrier frequency $\omega_{0}$, we observe an exponential decay of the direct waves

$$
I_{s}(t, \boldsymbol{x}) \stackrel{B \ll \omega_{0}}{\simeq} I_{\mathrm{hom}}(t, \boldsymbol{x}) \exp \left(-\frac{\sqrt{L^{2}+|\boldsymbol{x}|^{2}}}{L_{\mathrm{loc}}}\right) .
$$

Here $L_{\text {loc }}$ is the localization length defined by

$$
L_{\mathrm{loc}}=\frac{4 c_{0}^{2}}{\gamma_{0} \omega_{0}^{2}},
$$

which plays a central role in the wave localization phenomenon as we will see below.

2) $I_{c}$ is the contribution of the coda waves. It is a continuous contribution supported in $t \in\left[\sqrt{L^{2}+|\boldsymbol{x}|^{2}} / c_{0}, \infty\right)$. It correspond to the contributions of the trajectories of the jump process that jump at least once. In particular, for $\boldsymbol{x}=\mathbf{0}$ it is given by

$$
\begin{aligned}
I_{c}(t, \mathbf{0})= & \frac{\gamma_{0}}{32 \pi^{3} \zeta_{0}^{2} c_{0} t^{2}} \exp \left(-\frac{\sigma_{0} c_{0} t}{\zeta_{0}}\right) \int_{-L}^{0} d z \int \exp \left(-\frac{\gamma_{0} \omega^{2}(L+z)}{4 c_{0}^{2}}\right) \\
& \times \mathbb{E}\left[\delta\left(c_{0} t-2 \int_{z}^{0} N_{\omega, 0}\left(z^{\prime}\right) d z^{\prime}-L\right) \mid N_{\omega, 0}(z)=1\right] \omega^{4}\left|\hat{f}_{z}(\omega)\right|^{2} d \omega .
\end{aligned}
$$

As we will see in Proposition 7.5, in the case of strong scattering most of the received energy is carried by the coda waves and the power delay spread (the time duration of the received signal) can be very long.

6.3. A Thick Weakly Scattering and Statistically Homogeneous Layer. We assume that the background velocity is constant and that the medium in $(-L, 0)$ consists of two different regions $\left(-L,-z_{s}\right)$ and $\left(-z_{s}, 0\right)$. The dissipation and integrated covariance jump at the interface $z=-z_{s}$ :

$$
\sigma(z)=\left\{\begin{array}{l}
\sigma_{0} \text { if } z \in\left(-z_{s}, 0\right) \\
\sigma_{1} \text { if } z \in\left(-\infty,-z_{s}\right)
\end{array}, \quad \gamma(z)=\left\{\begin{array}{l}
\gamma_{0} \text { if } z \in\left(-z_{s}, 0\right) \\
0 \text { if } z \in\left(-\infty,-z_{s}\right)
\end{array} .\right.\right.
$$

Here $\sigma_{0}$ and $\sigma_{1}$ can be equal and we assume $\gamma_{0}>0$. Thus, we can in particular consider the situation when the interface only distinguishes itself via a jump in the scattering 
coefficient, that is, a change in the fluctuation magnitude and/or decoherence length of the microscale medium fluctuations from a situation with relatively small fluctuations. We assume that the top region is scattering which conforms with typical situations in the earth's crust with relative stronger scattering in the surface layer. We suppose that there is no impedance and background velocity contrast ( $\zeta$ and $c$ are constant and equal to $\zeta_{0}$ and $\left.c_{0}\right)$. Therefore, the interface $z=-z_{s}$ does not generate reflections itself, but the change in the scattering properties across the interface modifies the spectral density of the waves observed at the surface. We now describe the theoretical asymptotic behavior of the spectral density in this situation. The following lemma follows directly from (5.6) and (5.8).

LEMMA 6.1. If the top layer is weakly scattering $\left(\gamma_{0} \omega^{2} z_{s}\right) /\left(4 c_{0}^{2}\right) \ll 1$, then we have

$$
\mathcal{U}(\omega, \kappa, \xi)=\mathcal{U}_{s}(\omega, \kappa, \xi)+\mathcal{U}_{c}(\omega, \kappa, \xi)
$$

where the singular and continuous components are given by

$$
\begin{aligned}
& \mathcal{U}_{s}(\omega, \kappa, \xi)=\exp \left(-\frac{c_{0}(\kappa)\left[\sigma_{0} z_{s}+\sigma_{1}\left(L-z_{s}\right)\right]}{\zeta_{0} c_{0}}\right) \exp \left(-\frac{\gamma_{0} c_{0}^{2}(\kappa) \omega^{2}}{4 c_{0}^{4}} z_{s}\right) \delta\left(\xi-c_{0}(\kappa) L\right), \\
& \mathcal{U}_{c}(\omega, \kappa, \xi) \simeq \frac{\gamma_{0} c_{0}(\kappa) \omega^{2}}{8 c_{0}^{4}} \exp \left(-\frac{\sigma_{0} \xi+c_{0}(\kappa)\left(\sigma_{1}-\sigma_{0}\right)\left(L-z_{s}\right)}{\zeta_{0} c_{0}}\right) \mathbf{1}_{\left[L, L+2 z_{s}\right]}\left(\frac{\xi}{c_{0}(\kappa)}\right) .
\end{aligned}
$$

Substituting into (4.2), we find that the intensity of the direct waves is:

$$
\begin{aligned}
I_{s}(t, \boldsymbol{x})= & \frac{1}{8 \pi^{3} \zeta_{0}^{2} c_{0}^{4} t^{2}} \frac{L^{2}}{L^{2}+|\boldsymbol{x}|^{2}} \exp \left(-\frac{\sigma_{0} z_{s}+\sigma_{1}\left(L-z_{s}\right)}{\zeta_{0}} \frac{\sqrt{L^{2}+|\boldsymbol{x}|^{2}}}{L}\right) \\
& \times \delta\left(t-\frac{\sqrt{L^{2}+|\boldsymbol{x}|^{2}}}{c_{0}}\right) \\
& \times \int \exp \left(-\frac{\gamma_{0} \omega^{2}}{4 c_{0}^{2}} z_{s} \frac{L^{2}+|\boldsymbol{x}|^{2}}{L^{2}}\right)\left|\frac{L \hat{f}_{z}(\omega)+\boldsymbol{x} \cdot \hat{\boldsymbol{f}}_{\boldsymbol{x}}(\omega)}{\sqrt{L^{2}+|\boldsymbol{x}|^{2}}}\right|^{2} \omega^{2} d \omega
\end{aligned}
$$

and the intensity of the coda waves is of the form

$$
\begin{aligned}
I_{c}(t, \boldsymbol{x}) \simeq & \frac{1}{8 \pi^{3} \zeta_{0}^{2} c_{0}^{4} t^{2}} \exp \left(-\frac{\sigma_{0} c_{0} t}{\zeta_{0}}-\frac{\left(\sigma_{1}-\sigma_{0}\right)\left(L-z_{s}\right)}{\zeta_{0}} \frac{\sqrt{L^{2}+|\boldsymbol{x}|^{2}}}{L}\right) \\
& \times \mathbf{1}_{\left[\sqrt{L^{2}+|\boldsymbol{x}|^{2}}, \sqrt{\left.\left(L+2 z_{s}\right)^{2}+|\boldsymbol{x}|^{2}\right]}\right.}\left(c_{0} t\right) \\
& \times \frac{\gamma_{0}}{8 c_{0}} \int\left|\frac{\sqrt{c_{0}^{2} t^{2}-|\boldsymbol{x}|^{2}}}{c_{0} t} \hat{f}_{z}(\omega)+\frac{\boldsymbol{x}}{c_{0} t} \cdot \hat{\boldsymbol{f}}_{\boldsymbol{x}}(\omega)\right|^{2} \omega^{4} d \omega .
\end{aligned}
$$

We can see that the duration of the coda waves in this weakly scattering regime,

$$
\Delta T_{\text {coda }}=\frac{1}{c_{0}}\left(\sqrt{\left(L+2 z_{s}\right)^{2}+|\boldsymbol{x}|^{2}}-\sqrt{L^{2}+|\boldsymbol{x}|^{2}}\right),
$$

is directly related to the thickness of the scattering region and hence can be exploited in the context of estimation.

7. Surface Trapping Results. In this section we give the main results of the paper, that describe the waveguide features that can be observed with pressure release boundary conditions in the context of a heterogeneous medium. 
7.1. Mean Power Transmission Coefficient Conservation. In the absence of dissipation and medium fluctuations the power transmission coefficient $\left|\mathcal{T}_{\omega, \kappa}^{\varepsilon}\right|^{2}$ is equal to one. The surprising fact is that in a certain sense this is the case also in the situation with random medium fluctuations:

Proposition 7.1. In absence of dissipation, the mean power transmission coefficient is equal to one in the limit $\varepsilon \rightarrow 0$ :

$$
\lim _{\varepsilon \rightarrow 0} \mathbb{E}\left[\left|\mathcal{T}_{\omega, \kappa}^{\varepsilon}\right|^{2}\right]=1
$$

This results follows from the identity (5.5) which shows that the integral of the spectral density with respect to $\xi$ in the absence of dissipation is equal to one.

It is important to understand that this conservation relation is true only after statistical averaging. The power transmission coefficient $\left|\mathcal{T}_{\omega, \kappa}^{\varepsilon}\right|^{2}$ has in fact large fluctuations as shown by the next proposition (proved in Appendix E):

Proposition 7.2. In absence of dissipation, the variance of the power transmission coefficient $\operatorname{Var}\left(\left|\mathcal{T}_{\omega, \kappa}^{\varepsilon}\right|^{2}\right)=\mathbb{E}\left[\left|\mathcal{T}_{\omega, \kappa}^{\varepsilon}\right|^{4}\right]-\mathbb{E}\left[\left|\mathcal{T}_{\omega, \kappa}^{\varepsilon}\right|^{2}\right]^{2}$ has the limit:

$$
\lim _{\varepsilon \rightarrow 0} \operatorname{Var}\left(\left|\mathcal{T}_{\omega, \kappa}^{\varepsilon}\right|^{2}\right)=\frac{6}{5} \exp \left(\int_{-L}^{0} \frac{5 \gamma(z) c(\kappa, z)^{2} \omega^{2}}{2 c(z)^{4}} d z\right)-\frac{6}{5} .
$$

The conservation relation (7.1) deserves some comments. In the case of a nondissipative homogeneous medium $\gamma(z) \equiv 0$, we have $\left|\mathcal{T}_{\omega, \kappa}^{\varepsilon}\right|=1$ and the result is clear. In the case of a non-dissipative random medium this result is not obvious. In the case of a strongly scattering medium in $(-L, 0)$, the power transmission coefficient without the pressure release boundary conditions, that is $\left|T_{\omega, \kappa}^{\varepsilon}\right|^{2}$, is small. More precisely, it is exponentially small as a function of the thickness $L$, which is a manifestation of wave localization in three-dimensional randomly layered media [7, Chapter 14]. Therefore, only a small amount of the wave energy released by the source reaches the surface. However this small amount of energy is then trapped in the region close to the surface because of the pressure release boundary conditions at the surface $z=0$. The mechanism is the following one: the surface sends back the waves into the region $(-L, 0)$; the energy conservation equation $\left|R_{\omega, \kappa}^{\varepsilon}\right|^{2}+\left|T_{\omega, \kappa}^{\varepsilon}\right|^{2}=1$ (see (A.8)) indicates that the power reflection coefficient $\left|R_{\omega, \kappa}^{\varepsilon}\right|^{2}$ of the random region $(-L, 0)$ is close to one, so that the waves are reflected back to the surface by the random medium. This process repeats itself and generates a lot of multiples. The overall result is (7.1). This process looks like a waveguiding effect. A similar effect can be seen when a strong deterministic interface is present within the region $(-L, 0)$ and we describe this in Section 7.6. Finally, we note that the statistical average in (7.1) can be replaced by a frequency average. Indeed the results obtained in [7, Chapter 14] show that the transmission and reflection coefficients $T_{\omega, \kappa}^{\varepsilon}$ and $R_{\omega, \kappa}^{\varepsilon}$ have a decoherence frequency of the order of $\varepsilon$, and this property is inherited by the generalized transmission coefficient $\mathcal{T}_{\omega, \kappa}^{\varepsilon}$. This decoherence property ensures that the average over a bandwidth of order one of the power transmission coefficient $\left|\mathcal{T}_{\omega, \kappa}^{\varepsilon}\right|^{2}$ is equal to one in the limit $\varepsilon \rightarrow 0$ in probability.

7.2. Invariance of Total Surface Energy. We here consider, in the nondissipative case, the total energy transmitted to the surface defined by:

$$
\mathcal{E}^{\varepsilon}=\frac{\zeta(0)}{2} \iint s u^{\varepsilon}(t, \boldsymbol{x})^{2} d \boldsymbol{x} d t
$$


Proposition 7.3. In absence of dissipation the total surface energy is conserved in the limit $\varepsilon \rightarrow 0$ :

$$
\mathcal{E}^{\varepsilon} \stackrel{\varepsilon \rightarrow 0}{\longrightarrow} \mathcal{E}_{0}, \quad \text { in probability, }
$$

where $\mathcal{E}_{0}$ is the total surface energy obtained in absence of random fluctuations:

$$
\mathcal{E}_{0}=\frac{\zeta(0)}{2(2 \pi)^{3} \rho(-L)^{2}} \iint\left|\frac{\hat{f}_{z}(\omega)}{c(\kappa,-L)}+\boldsymbol{\kappa} \cdot \hat{\boldsymbol{f}}_{\boldsymbol{x}}(\omega)\right|^{2} \frac{\zeta(\kappa,-L)}{\zeta(\kappa, 0)} \omega^{2} d \boldsymbol{\kappa} d \omega .
$$

This proposition shows that the total surface energy does not depend on the scattering medium model in the small $\varepsilon$ limit, moreover, the statistical fluctuations in the total surface energy vanishes in this limit. The conservation of the total surface energy is remarkable and follows from the pressure release boundary conditions. We will discuss this further in the next subsection.

Proof. Using (3.2) and Parseval's formula we find

$$
\mathcal{E}^{\varepsilon}=\frac{\zeta(0)}{2(2 \pi)^{3}} \iint\left|\hat{g}^{\varepsilon}(\omega, \boldsymbol{\kappa})\right|^{2}\left|\mathcal{T}_{\omega, \kappa}^{\varepsilon}\right|^{2} \omega^{2} d \boldsymbol{\kappa} d \omega .
$$

From (7.1) it follows

$$
\lim _{\varepsilon \rightarrow 0} \mathbb{E}\left[\mathcal{E}^{\varepsilon}\right]=\mathcal{E}_{0}
$$

This result gives the conservation of the mean total surface energy. Moreover, this quantity is self-averaging. Indeed, we have

$$
\left(\mathcal{E}^{\varepsilon}\right)^{2}=\frac{\zeta(0)^{2}}{4(2 \pi)^{6}} \iint\left|\hat{g}^{\varepsilon}\left(\omega_{1}, \boldsymbol{\kappa}_{1}\right)\right|^{2}\left|\hat{g}^{\varepsilon}\left(\omega_{2}, \boldsymbol{\kappa}_{2}\right)\right|^{2}\left|\mathcal{T}_{\omega_{1}, \kappa_{1}}^{\varepsilon}\right|^{2}\left|\mathcal{T}_{\omega_{2}, \kappa_{2}}^{\varepsilon}\right|^{2} \omega_{1}^{2} \omega_{2}^{2} d \boldsymbol{\kappa}_{1} d \omega_{1} d \boldsymbol{\kappa}_{2} d \omega_{2},
$$

and by an argument that parallels the one presented in Appendix D, exploiting the decoherence properties of the generalized transmission coefficient:

$$
\lim _{\varepsilon \rightarrow 0}\left|\mathbb{E}\left[\left|\mathcal{T}_{\omega_{1}, \kappa_{1}}^{\varepsilon}\right|^{2}\left|\mathcal{T}_{\omega_{2}, \kappa_{2}}^{\varepsilon}\right|^{2}\right]-\mathbb{E}\left[\left|\mathcal{T}_{\omega_{1}, \kappa_{1}}^{\varepsilon}\right|^{2}\right] \mathbb{E}\left[\left|\mathcal{T}_{\omega_{2}, \kappa_{2}}^{\varepsilon}\right|^{2}\right]\right|=0, \quad \text { for } \omega_{1} \neq \omega_{2}
$$

we find

$$
\lim _{\varepsilon \rightarrow 0} \mathbb{E}\left[\left(\mathcal{E}^{\varepsilon}\right)^{2}\right]=\mathcal{E}_{0}^{2}
$$

Therefore, the variance of $\mathcal{E}^{\varepsilon}$ goes to zero as $\varepsilon \rightarrow 0$ which gives the desired result.

7.3. Mean Surface Energy Density Conservation. When the medium is random, non-dissipative, and with constant background velocity $c_{0}$ and impedance $\zeta_{0}$, the mean transmitted intensity received at $\boldsymbol{x}=\mathbf{0}$ can be expressed using (4.2) as:

$$
I(t, \mathbf{0})=\frac{1}{(2 \pi)^{3} c_{0}^{2} \zeta_{0}^{2} t^{2}} \int \mathcal{U}\left(\omega, 0, c_{0}^{2} t\right)\left|\hat{f}_{z}(\omega)\right|^{2} \omega^{2} d \omega
$$

If we compensate for the algebraic decay due to geometric spreading, then using the fact that $\int \mathcal{U}(\omega, \kappa, \xi) d \xi=1$ (see (5.5)) we find the following energy conservation property.

Proposition 7.4. In absence of dissipation and with a constant background the "modified" surface energy density at $\boldsymbol{x}=\mathbf{0}$ does not depend on scattering:

$$
\int t^{2} I(t, \mathbf{0}) d t=\int t^{2} I_{\mathrm{hom}}(t, \mathbf{0}) d t,
$$


where the right-hand side is given by (6.2).

The "modified" surface energy density thus coincides with the one in a homogeneous medium. The conservation of the "modified" surface energy density is quite remarkable and follows from the pressure release boundary conditions. Indeed, in the case of matched boundary conditions at the surface and with a constant scattering coefficient $\gamma_{0}$, we have $[7$, Chapter 14]

$$
\left.\int t^{2} I(t, \mathbf{0}) d t\right|_{\mathrm{MBC}}=\frac{1}{(2 \pi)^{3} c_{0}^{4} \zeta_{0}^{2}} \int \Xi_{1}\left(\frac{\gamma_{0} \omega^{2}}{4 c_{0}^{2}} L\right)\left|\hat{f}_{z}(\omega)\right|^{2} \omega^{2} d \omega,
$$

with

$$
\Xi_{1}(\Gamma)=\exp \left(-\frac{\Gamma}{4}\right) \int_{0}^{\infty} e^{-\mu^{2} \Gamma} \frac{2 \pi \mu \sinh (\mu \pi)}{\cosh ^{2}(\mu \pi)} d \mu \stackrel{\Gamma \geqq 1}{\simeq} \frac{\pi^{5 / 2}}{2 \Gamma^{3 / 2}} \exp \left(-\frac{\Gamma}{4}\right)
$$

Therefore, with matched boundary conditions and with a pulse source with small bandwidth $B$ and carrier frequency $\omega_{0}$, we have

$$
\left.\int t^{2} I(t, \mathbf{0}) d t\right|_{\mathrm{MBC}} \stackrel{B \ll \omega_{0}}{\simeq}\left[\int t^{2} I_{\mathrm{hom}}(t, \mathbf{0}) d t\right] \Xi_{1}\left(\frac{L}{L_{\mathrm{loc}}}\right)
$$

where the localization length $L_{\mathrm{loc}}$ is defined by (6.7). This exhibits the exponential decay of the transmitted energy associated with the exponential localization of waves. This exponential decay is not observed in Eq. (7.8), which deserves some explanations:

When scattering is weak (in the sense that $L / L_{\mathrm{loc}} \ll 1$ ), the transmission coefficient $\left|T_{\omega, \kappa}^{\varepsilon}\right|$ of the random section $(-L, 0)$ is close to one, and the "modified" surface energy can indeed be expected to be close to the emitted energy. This holds true for the matched boundary conditions and for the pressure release boundary conditions.

When scattering is strong (in the sense that $L / L_{\mathrm{loc}}>1$ ), the transmission coefficient $\left|T_{\omega, \kappa}^{\varepsilon}\right|$ of the random section $(-L, 0)$ is close to zero. That is why the "modified" surface energy is small in the case of matched boundary conditions since only a very small fraction of the wave energy reaches the surface. However, with pressure release boundary conditions, the waves that reach the surface are sent back into the medium. With strong scattering, the reflection coefficient $\left|R_{\omega, \kappa}^{\varepsilon}\right|$ of the random section $(-L, 0)$ is close to one, so the waves are reflected back by the medium to the surface. This is repeated many times and the waves are reflected back and forth between the surface and the random medium. We observe again that the waves are trapped in this waveguide and the overall result in terms of the energy received at the surface is (7.8). Of course the multiples are received after a long delay, which means that the power delay spread is long. We analyze the power delay spread in the next subsection.

7.4. Coda Enhancement. In order to study the power delay spread we look at the mean reception time of the "modified" surface energy density at $\boldsymbol{x}=\mathbf{0}$ :

$$
\langle t\rangle:=\frac{\int t^{3} I(t, \mathbf{0}) d t}{\int t^{2} I(t, \mathbf{0}) d t}
$$

To characterize this quantity we again look at the situation with a constant background medium. Using Appendix F we obtain the following proposition.

Proposition 7.5. Let us consider a source pulse with small bandwidth $B$ and carrier frequency $\omega_{0}$, and denote by $L_{\mathrm{loc}}$ the associated localization length (6.7). 
1) When scattering is weak, in the sense that $L / L_{\mathrm{loc}} \ll 1$, then the correction to the deterministic travel time $L / c_{0}$ is small and given by

$$
\langle t\rangle \stackrel{B \ll \omega_{0}, L \gg L_{\mathrm{loc}}}{\simeq} \frac{L}{c_{0}}\left[1+\frac{L}{L_{\mathrm{loc}}}+O\left(\frac{L^{2}}{L_{\mathrm{loc}}^{2}}\right)\right] .
$$

2) When scattering is strong, in the sense that $L / L_{\mathrm{loc}} \gg 1$, then

$$
\langle t\rangle \stackrel{B \ll \omega_{0}, L \gg L_{\mathrm{loc}}}{\simeq} \frac{2 c_{0}}{\gamma_{0} \omega_{0}^{2}} \exp \left(\frac{\gamma_{0} \omega_{0}^{2}}{2 c_{0}^{2}} L\right)=\frac{L_{\mathrm{loc}}}{2 c_{0}} \exp \left(\frac{2 L}{L_{\mathrm{loc}}}\right) .
$$

The result (7.10) is in agreement with the fact that in this case most of the energy is carried by the direct waves that are received at the deterministic travel time $L / c_{0}$, while the result (7.11) shows that the coda waves (and the power delay spread) are very long and most of the energy is carried by the coda waves in the case with strong medium fluctuations. This delay spread behavior is a striking and important aspect of the pressure release boundary conditions.

7.5. Incoherent Interface Trapping. We assume here that the medium has constant background velocity, impedance, and dissipation (denoted by $c_{0}, \zeta_{0}$, and $\sigma_{0}$ ) and that it is homogeneous except in a layer located in $z \in\left(-z_{0},-z_{0}+\Delta z\right)$ in which it is scattering. The integrated covariance has the form

$$
\gamma(z)=\left\{\begin{array}{l}
0 \text { if } z \in\left(-z_{0}+\Delta z, 0\right) \\
\gamma_{0} \text { if } z \in\left(-z_{0},-z_{0}+\Delta z\right] \\
0 \text { if } z \in\left(-\infty,-z_{0}\right]
\end{array}\right.
$$

with $-z_{0} \in(-L, 0)$. In the limit case of a thin and strong scattering layer:

$$
\Delta z \rightarrow 0 \text { and } \gamma_{0} \Delta z \rightarrow \widetilde{\gamma_{0}}>0
$$

we obtain in Appendix G the following result.

Proposition 7.6. The mean intensity at the surface at $(\boldsymbol{x}, 0)$ is of the form:

$$
I(t, \boldsymbol{x})=\frac{S_{n} O^{2}}{(2 \pi)^{3} \zeta_{0}^{2} c_{0}^{2} S_{n} M^{4}} \sum_{n=0}^{\infty} \delta\left(t-\frac{S_{n} M}{c_{0}}\right) \int \alpha_{n}\left(\omega, \kappa_{n}\right)\left|\frac{\overrightarrow{S_{n} M}}{S_{n} M} \cdot \hat{\overrightarrow{\boldsymbol{f}}}(\omega)\right|^{2} \omega^{2} d \omega
$$

with $c_{0} \kappa_{n}=|\boldsymbol{x}| / S_{n} M, S_{n}=\left(\mathbf{0},-L-2 n z_{0}\right)$ is the position of a virtual source, $O=$ $(\mathbf{0}, 0)$ is the position of the point at the surface just above the source, and $M=(\boldsymbol{x}, 0)$ is the position of the observation point at the surface. For $\boldsymbol{x}=\mathbf{0}$ one has:

$$
I(t, \mathbf{0})=\frac{1}{(2 \pi)^{3} \zeta_{0}^{2} c_{0}^{4} t^{2}} \sum_{n=0}^{\infty} \delta\left(t-\frac{L+2 n z_{0}}{c_{0}}\right) \int \alpha_{n}(\omega, 0)\left|\hat{f}_{z}(\omega)\right|^{2} \omega^{2} d \omega .
$$

The amplitudes $\alpha_{n}(\omega, \kappa)$ of the multiples are monotoneously decaying in $n$ and they are given by

$$
\alpha_{n}(\omega, \kappa)=\sum_{j=0}^{n}\left(\begin{array}{l}
n \\
j
\end{array}\right)(-1)^{j} \Xi_{j+1}\left(\Gamma_{\omega, \kappa}\right), \quad \Gamma_{\omega, \kappa}=\frac{\widetilde{\gamma_{0}} \omega^{2} c_{0}^{2}(\kappa)}{4 c_{0}^{4}},
$$

where

$$
\Xi_{n}(\Gamma)=\exp \left(-\frac{\Gamma}{4}\right) \int_{0}^{\infty} e^{-\mu^{2} \Gamma} \frac{2 \pi \mu \sinh (\mu \pi)}{\cosh ^{2}(\mu \pi)} K^{(n)}(\mu) d \mu,
$$



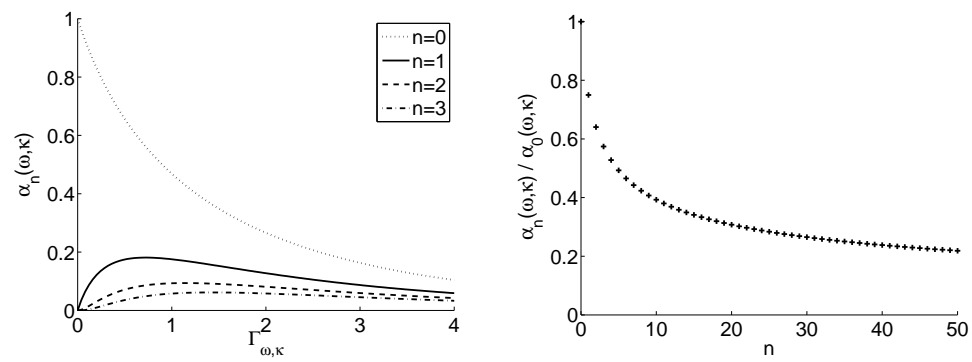

FIG. 7.1. Left figure: the function $\alpha_{n}(\omega, \kappa)$ as a function of $\Gamma_{\omega, \kappa}$ for different $n$. Right figure: the function $\alpha_{n}(\omega, \kappa) / \alpha_{0}(\omega, \kappa)$ as a function of $n$ in the limit $\Gamma_{\omega, \kappa} \gg 1$.

and

$$
K^{(1)}(\mu)=1, \quad K^{(n)}(\mu)=\prod_{j=1}^{n-1} \frac{1}{j^{2}}\left[\mu^{2}+\left(j-\frac{1}{2}\right)^{2}\right] \text { for } n \geq 2 .
$$

We remark that:

1) The $n$-th multiple is not a short pulse of the same form as the source pulse, but it is an incoherent wave packet that is localized in time around the arrival time $S_{n} M / c_{0}$ which corresponds to the travel time of a wave transmitted through the thin scattering layer and reflected back and forth $n$ times between the surface and the scattering layer. 2) The decay of the amplitudes of the multiples is not exponential, as it would have been with a strong interface, see the next subsection. This is characteristic of a scattering layer. As we now show, for a strongly incoherent scattering layer, the decay can be very slow.

In the weakly scattering regime $\Gamma_{n, \kappa} \ll 1$ we have for any $n \geq 0$ :

$$
\alpha_{n}(\omega, \kappa) \stackrel{\Gamma_{\omega, \kappa} \ll 1}{\simeq} n ! \Gamma_{\omega, \kappa}^{n}\left(1+O\left(\Gamma_{\omega, \kappa}\right)\right) .
$$

This result (which holds as long as $n^{2} \Gamma_{\omega, \kappa} \ll 1$ ) shows that the exponential decay of the amplitudes of the multiples observed for a deterministic interface is not valid anymore. This phenomenon is even more apparent in the strongly scattering regime $\Gamma_{\omega, \kappa} \gg 1$. The asymptotic behavior of the function $\Xi_{n}(\Gamma)$ for large $\Gamma$ is

$$
\Xi_{n}(\Gamma) \stackrel{\Gamma \gg 1}{\simeq} \frac{\pi^{5 / 2} K^{(n)}(0)}{2 \Gamma^{3 / 2}} \exp \left(-\frac{\Gamma}{4}\right),
$$

so that, for a strongly scattering thin layer, we have for $n \geq 1$,

$$
\frac{\alpha_{n}(\omega, \kappa)}{\alpha_{0}(\omega, \kappa)} \stackrel{\Gamma_{\omega, \kappa} \gg 1}{\simeq} 1+\sum_{j=1}^{n}\left(\begin{array}{c}
n \\
j
\end{array}\right)(-1)^{j} \prod_{j^{\prime}=1}^{j}\left(1-\frac{1}{2 j^{\prime}}\right)^{2} .
$$

The amplitudes of the multiples $\alpha_{n}(\omega, \kappa)$ are plotted as functions of $n$ and $\Gamma_{\omega, \kappa}$ in Figure 7.1. The slow decay in $n$ can be observed.

7.6. Strong Interface Trapping. In this section we depart from the situation with a smooth background medium modulated by random fluctuations, which is our 
main focus, and discuss the case of a background medium with a strong and deterministic interface and without random fluctuations. This allows us to contrast the wave trapping seen in a random medium with the one obtained in a homogeneous medium and generated by a jump in the background. We assume thus that the density, bulk modulus, and dissipation of the medium are stepwise constant:

$$
\begin{aligned}
K^{\varepsilon-1}(z) & =\left\{\begin{array}{ll}
K_{0}^{-1} & \text { if } z \in\left(-\infty,-z_{i}\right) \\
K_{1}^{-1} & \text { if } z \in\left(-z_{i}, 0\right)
\end{array},\right. \\
\rho^{\varepsilon}(z) & =\left\{\begin{array}{ll}
\rho_{0} & \text { if } z \in\left(-\infty,-z_{i}\right) \\
\rho_{1} & \text { if } z \in\left(-z_{i}, 0\right)
\end{array},\right. \\
\sigma^{\varepsilon}(z) & = \begin{cases}\sigma_{0} & \text { if } z \in\left(-\infty,-z_{i}\right) \\
\sigma_{1} & \text { if } z \in\left(-z_{i}, 0\right)\end{cases}
\end{aligned}
$$

giving a jump in the impedance at the interface $z=-z_{i}$, where $-z_{i} \in(-L, 0)$. The vertical velocity field recorded at the surface $z=0$ has the integral representation

$$
\begin{array}{r}
u^{\varepsilon}(t, \boldsymbol{x})=\frac{1}{(2 \pi)^{3} \varepsilon^{3 / 2}} \iint\left[\frac{\hat{f}_{z}^{\varepsilon}(\omega)}{\zeta_{0}^{1 / 2}(\kappa) \zeta_{1}^{1 / 2}(\kappa)}+\frac{i \omega}{i \omega \rho_{0}-\varepsilon \sigma_{0}} \frac{\zeta_{0}^{1 / 2}(\kappa)}{\zeta_{1}^{1 / 2}(\kappa)} \boldsymbol{\kappa} \cdot \hat{\boldsymbol{f}}_{\boldsymbol{x}}^{\varepsilon}(\omega)\right] \\
\times \mathcal{T}_{\omega, \kappa}^{\varepsilon} \exp \left(-i \frac{\omega}{\varepsilon}(t-\boldsymbol{\kappa} \cdot \boldsymbol{x})\right) \omega^{2} d \boldsymbol{\kappa} d \omega \cdot(7.16)
\end{array}
$$

The generalized transmission coefficient has now the form

$$
\begin{aligned}
\mathcal{T}_{\omega, \kappa}^{\varepsilon}= & T_{i}(\kappa) \exp \left(\left(\frac{i \omega}{\varepsilon c_{0}(\kappa)}-\frac{\sigma_{0} c_{0}(\kappa)}{2 \zeta_{0} c_{0}}\right)\left(L-z_{i}\right)\right) \exp \left(\left(\frac{i \omega}{\varepsilon c_{1}(\kappa)}-\frac{\sigma_{1} c_{1}(\kappa)}{2 \zeta_{1} c_{1}}\right) z_{i}\right) \\
& \times\left[1-R_{i}(\kappa) \exp \left(2\left(\frac{i \omega}{\varepsilon c_{1}(\kappa)}-\frac{\sigma_{1} c_{1}(\kappa)}{2 \zeta_{1} c_{1}}\right) z_{i}\right)\right]^{-1}
\end{aligned}
$$

where

$$
R_{i}(\kappa)=\frac{\zeta_{1}(\kappa)-\zeta_{0}(\kappa)}{\zeta_{1}(\kappa)+\zeta_{0}(\kappa)} \quad \text { and } \quad T_{i}(\kappa)=\frac{2 \sqrt{\zeta_{1}(\kappa) \zeta_{0}(\kappa)}}{\zeta_{1}(\kappa)+\zeta_{0}(\kappa)}
$$

are the mode-dependent reflection and transmission coefficients of the interface at $z=-z_{i}$. The generalized transmission coefficient can be expanded as

$$
\begin{aligned}
\mathcal{T}_{\omega, \kappa}^{\varepsilon}= & T_{i}(\kappa) \exp \left(\left(\frac{i \omega}{\varepsilon c_{0}(\kappa)}-\frac{\sigma_{0} c_{0}(\kappa)}{2 \zeta_{0} c_{0}}\right)\left(L-z_{i}\right)\right) \\
& \times \sum_{j=0}^{\infty} R_{i}^{j}(\kappa) \exp \left((2 j+1)\left(\frac{i \omega}{\varepsilon c_{1}(\kappa)}-\frac{\sigma_{1} c_{1}(\kappa)}{2 \zeta_{1} c_{1}}\right) z_{i}\right) .
\end{aligned}
$$

Each term of the sum can be interpreted as the contribution of the waves transmitted though the interface and then reflected back and forth $j$ times between the surface $z=0$ and the interface $z=-z_{i}$.

We next consider the vertical velocity field at the position $O=(\mathbf{0}, 0)$ in the limit $\varepsilon \rightarrow 0$. We apply the stationary phase method and find that the value of the integral (7.16) for the $j$-th multiple is given by the contribution around the stationary slowness vector $\boldsymbol{\kappa}_{\mathrm{sp}, j}=\mathbf{0}$ and is concentrated around the time

$$
t_{\mathrm{sp}, j}=\frac{L-z_{i}}{c_{0}}+\frac{(2 j+1) z_{i}}{c_{1}}
$$


which is the travel time of the ray going from the source $S=(\mathbf{0},-L)$ to the observation point $O=(\mathbf{0}, 0)$ after $j$ reflections between the surface $z=0$ and the interface $z=-z_{i}$. Finally, we find that the wave is transmitted in the form of train of short pulses that are received at the travel times $\left\{t_{\mathrm{sp}, j}, j \in \mathbb{N}\right\}$ and we have for any $j \in \mathbb{N}$ :

$$
\begin{aligned}
\lim _{\varepsilon \rightarrow 0} \varepsilon^{1 / 2} u^{\varepsilon}\left(t_{\mathrm{sp}, j}+\varepsilon s, \mathbf{0}\right)= & \frac{T_{i}(0) R_{i}^{j}(0)}{2 \pi \zeta_{0}^{1 / 2} \zeta_{1}^{1 / 2}\left[c_{0}\left(L-z_{i}\right)+(2 j+1) c_{1} z_{i}\right]} \\
& \times \exp \left(-\frac{\sigma_{0}\left(L-z_{i}\right)}{2 \zeta_{0}}-\frac{(2 j+1) \sigma_{1} z_{i}}{2 \zeta_{1}}\right) f_{z}^{\prime}(s) .
\end{aligned}
$$

Beyond the power law decay due to the geometric spreading and the exponential decay due to dissipation, the exponential decay of the energies carried by the multiples can be observed due to the factor $R_{i}^{j}(0)$. This exponential decay is typical of a deterministic interface.

We now highlight that some of the particular features of the surface wave energy that originate from the pressure release boundary conditions, and which we have discussed in the context of a random medium, is also present in the case with a deterministic interface. In absence of dissipation the power transmission coefficient $\left|\mathcal{T}_{\omega, \kappa}^{\varepsilon}\right|^{2}$ is given by

$$
\left|\mathcal{T}_{\omega, \kappa}^{\varepsilon}\right|^{2}=\frac{1-R_{i}^{2}(\kappa)}{\left|1-R_{i}(\kappa) \exp \left(2 i \frac{\omega}{\varepsilon c_{1}(\kappa)} z_{i}\right)\right|^{2}},
$$

where we have used the relation $T_{i}^{2}(\kappa)=1-R_{i}^{2}(\kappa)$. For a given slowness $\kappa$, the power transmission coefficient displays an interesting behavior as a function of the frequency $\omega$. It oscillates periodically between the two extremal values

$$
\min _{\omega}\left|\mathcal{T}_{\omega, \kappa}^{\varepsilon}\right|^{2}=\frac{1-\left|R_{i}(\kappa)\right|}{1+\left|R_{i}(\kappa)\right|}, \quad \max _{\omega}\left|\mathcal{T}_{\omega, \kappa}^{\varepsilon}\right|^{2}=\frac{1+\left|R_{i}(\kappa)\right|}{1-\left|R_{i}(\kappa)\right|},
$$

with the small period $\omega_{p}=\varepsilon \pi c_{1}(\kappa) / z_{i}$. The interesting point is that its frequencyaverage is equal to one whatever the value of the reflectivity of the interface $R_{i}(\kappa)$ :

$$
\left\langle\left|\mathcal{T}_{\omega, \kappa}^{\varepsilon}\right|^{2}\right\rangle_{\omega}=1 \text {. }
$$

Note that this conservation relation is valid only after frequency averaging, and that the variations of the transmission coefficient within the period are large when the interface is strong $\left|R_{i}(\kappa)\right| \sim 1$ :

$$
\left\langle\left|\mathcal{T}_{\omega, \kappa}^{\varepsilon}\right|^{4}\right\rangle_{\omega}=\frac{1+R_{i}^{2}(\kappa)}{1-R_{i}^{2}(\kappa)} .
$$

Eq. (7.18) shows that, if we compensate for dissipation, then the total energy received at the surface and emitted by a broadband source such as (2.6) does not depend on the reflectivity of the interface. With a weak interface $\left|R_{i}(\kappa)\right| \ll 1$, most of the energy is carried by the direct waves (the term $j=0$ ) which have strong amplitudes. With a strong interface $\left|R_{i}(\kappa)\right| \sim 1$, the energy is distributed over a long train of short pulses which have small and exponentially decaying amplitudes. Therefore, although the energy distribution amongst the multiples depends strongly on the interface reflectivity, the total received energy does not. This is characteristic of the pressure release boundary conditions and a similar phenomenon was observed in the case of a randomly scattering medium in Subsections 7.1-7.3. 
8. Numerical simulations. In this section we present results of numerical simulations that illustrate some of the theoretical predictions obtained in this paper.

We consider the case of a statistically homogeneous and non-dissipative medium. The medium in $[-L, 0]$ is the concatenation of thin layers with constant density and alternating bulk modulus with values $K=K_{0} /\left(1 \pm \sigma_{K}\right)$. We address two different values for $\sigma_{K}: \sigma_{K}=0.8$ (simulation a) and $\sigma_{K}=0.4$ (simulation b). The background density $\rho_{0}$, bulk modulus $K_{0}$, and speed of sound $c_{0}$ are all equal to one. The thicknesses of the layers are independent exponential random variables with mean $l_{c}=0.05$ (simulation a) and $l_{c}=0.2$ (simulation $\mathrm{b}$ ). The integrated covariance is $\gamma_{0}=\sigma_{K}^{2} l_{c}=0.032$ in cases a and b. The total thickness of the random slab is $L=100$ and the point source is located at $(-L, \mathbf{0})$. The source pulse emits in the vertical direction and has the form of the second derivative of a Gaussian, with Fourier transform $\hat{f}_{z}(\omega)=\omega^{2} \exp \left(-\omega^{2}\right)$. The carrier (angular) frequency is $\omega_{0}=1$. The localization length is $L_{\mathrm{loc}}=4 c_{0}^{2} /\left(\gamma_{0} \omega_{0}^{2}\right)=125$ for the carrier frequency $\omega_{0}$, which means that the localization length $L_{\mathrm{loc}}$ is here of the order of the propagation distance $L$.

We consider the time-dependent surface intensity $u^{2}(t, \mathbf{0})$. On the one hand we plot in Fig. 8.1 the theoretical asymptotic formula (7.7) with the power spectral density obtained by Monte Carlo simulations of the probabilistic representation (6.3). On the other hand we perform a series of 100 full numerical simulations of the wave equation with independent realizations of the random medium in cases a and b, and we plot the numerically averaged time-dependent surface intensity. The agreement between the numerical averages and the theoretical asymptotic formulas shows that the asymptotic theory is valid in this example in which the separation of scales is not so large $(\varepsilon \sim 0.1)$. Note that in Fig. 8.1 we also plot the time-dependent surface intensity for one realization, which indeed is not a statistically stable function. It is also clear from the figure that we have a long power delay spread, consistently with Proposition 7.5.

We also evaluate for each simulation the total surface energy $\iint u^{2}(t, \boldsymbol{x}) d t d \boldsymbol{x}$ and the "modified" surface energy density $\int t^{2} u^{2}(t, \mathbf{0}) d t$. We plot in Figs. 8.2-8.3 the histograms of the 100 total surface energies and "modified" surface energy densities obtained with the independent realizations of the random medium. From the asymptotic theory, we can expect that these quantities should not depend on scattering and that they should be equal to $\iint I_{\mathrm{hom}}(t, \boldsymbol{x}) d t d \boldsymbol{x}$ and $\int t^{2} I_{\mathrm{hom}}(t, \mathbf{0}) d t$, respectively, which is indeed what is observed in the numerical simulations, although fluctuations of the order of $\varepsilon$ are still noticeable.

Finally, note that we have performed two series of 100 simulations, in cases a and $\mathrm{b}$, respectively, with two sets of parameters $\left(l_{c}=0.2, \sigma_{K}=0.4\right)$ and $\left(l_{c}=0.05, \sigma_{K}=\right.$ $0.8)$. The parameter $\gamma_{0}$ is the same in cases a and b, so that the theoretical asymptotic formulas are unchanged. It can be seen in Figs. 8.1-8.3 that the numerical results obtained in cases a and $\mathrm{b}$ are indeed similar.

9. Conclusion. In this paper we have analyzed the propagation of waves generated by a point source located in a randomly layered medium in the presence of pressure release boundary conditions at the surface. We have found conserved quantities specific to pressure release boundary conditions that are different from the ones obtained with matched boundary conditions. We have identified a probabilistic representation for the spectral density of the waves received at the surface in terms of a jump Markov process. We have seen that the power delay spread can be very long due to the multiple reflections between the surface and the random medium. This waveguiding effect is quite different from the one observed in a standard deterministic 
a)

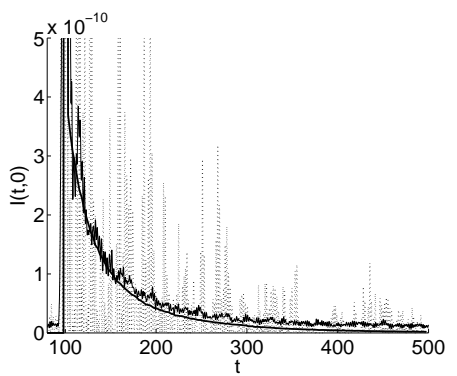

b)

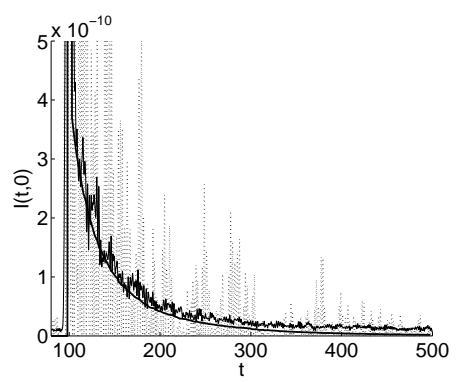

FIG. 8.1. The theoretical mean transmitted intensity (thick solid line), the transmitted intensity for a particular realization of the medium (dotted line), and the transmitted intensity averaged over 100 realizations of the random medium (thin solid line). In figure $a, l_{c}=0.05$ and $\sigma_{K}=0.8$. In figure $b, l_{c}=0.2$ and $\sigma_{K}=0.4$.

a)

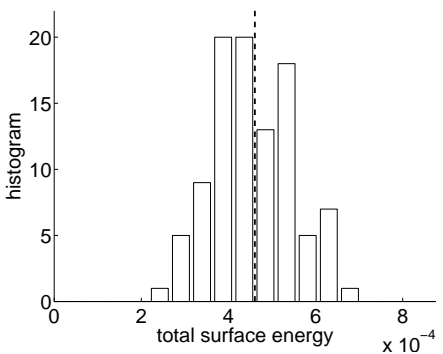

b)

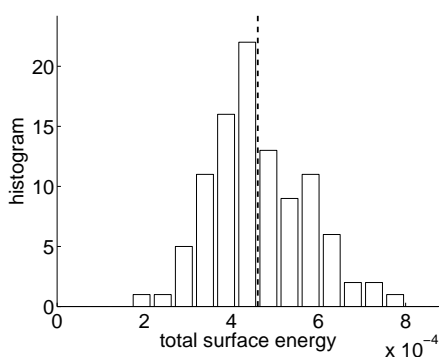

FIG. 8.2. Histogram of the total surface energies obtained with 100 independent simulations. The dashed line is the total surface energy obtained with a homogeneous medium. In figure a, $l_{c}=0.05$ and $\sigma_{K}=0.8$. In figure $b, l_{c}=0.2$ and $\sigma_{K}=0.4$.

waveguide in that the decay of the intensity is not exponential.

The results of this paper have been obtained for randomly layered media, however, we anticipate that they are robust and can be extended qualitatively to locally layered media as studied in $[14,16]$. Other propagation regimes, such as the radiative transport regime [12], or the parabolic regime [10], deserve specific analysis.

Appendix A. Derivation of the Integral Representation of the Recorded Field. We consider the general model (2.2-2.4) for the parameters of the random medium. We use the same approach as in [7] applying a propagator formulation. We first take a scaled Fourier transform in time and transverse spatial coordinates:

$$
\begin{aligned}
& \hat{p}^{\varepsilon}(\omega, \boldsymbol{\kappa}, z)=\iint p^{\varepsilon}(t, \boldsymbol{x}, z) e^{i \frac{\omega}{\varepsilon}(t-\boldsymbol{\kappa} \cdot \boldsymbol{x})} d t d \boldsymbol{x}, \\
& \hat{u}^{\varepsilon}(\omega, \boldsymbol{\kappa}, z)=\iint u^{\varepsilon}(t, \boldsymbol{x}, z) e^{i \frac{\omega}{\varepsilon}(t-\boldsymbol{\kappa} \cdot \boldsymbol{x})} d t d \boldsymbol{x} .
\end{aligned}
$$

We introduce the right- and left-going modes defined by

$$
\begin{aligned}
& \hat{a}^{\varepsilon}(\omega, \kappa, z)=\left(\frac{1}{\sqrt{\zeta(\kappa, z)}} \hat{p}^{\varepsilon}(\omega, \kappa, z)+\sqrt{\zeta(\kappa, z)} \hat{u}^{\varepsilon}(\omega, \kappa, z)\right) e^{-i \frac{\omega}{\varepsilon} \tau(\kappa, z)}, \\
& \hat{b}^{\varepsilon}(\omega, \kappa, z)=\left(-\frac{1}{\sqrt{\zeta(\kappa, z)}} \hat{p}^{\varepsilon}(\omega, \kappa, z)+\sqrt{\zeta(\kappa, z)} \hat{u}^{\varepsilon}(\omega, \kappa, z)\right) e^{i \frac{\omega}{\varepsilon} \tau(\kappa, z)} .
\end{aligned}
$$


a)

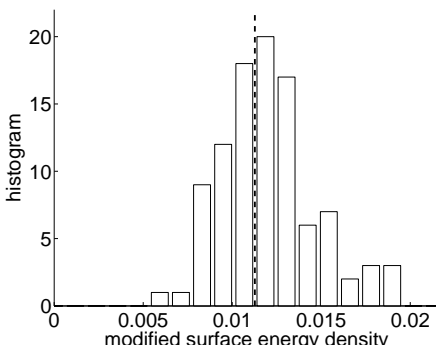

b)

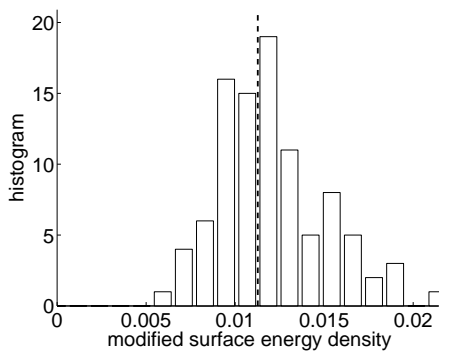

FIG. 8.3. Histogram of the "modified" surface energy densities obtained with 100 independent simulations. The dashed line is the "modified" surface energy density obtained with a homogeneous medium. In figure $a, l_{c}=0.05$ and $\sigma_{K}=0.8$. In figure $b, l_{c}=0.2$ and $\sigma_{K}=0.4$.

The pressure release boundary conditions at the surface $z=0$ is $\hat{p}^{\varepsilon}=0$, or equivalently

$$
\hat{a}^{\varepsilon}(\omega, \kappa, 0)=\hat{b}^{\varepsilon}(\omega, \kappa, 0) .
$$

The jump conditions across the source plane at $z=-L$ are

$$
\left[\hat{p}^{\varepsilon}\right]_{(-L)^{-}}^{(-L)^{+}}=\varepsilon^{3 / 2} \hat{f}_{z}(\omega), \quad\left[\hat{u}^{\varepsilon}\right]_{(-L)^{-}}^{(-L)^{+}}=\frac{i \varepsilon^{3 / 2} \omega}{\rho(-L) i \omega-\varepsilon \sigma(-L)} \boldsymbol{\kappa} \cdot \hat{\boldsymbol{f}}_{\boldsymbol{x}}(\omega)
$$

which give with the radiation condition $\hat{a}^{\varepsilon}\left(\omega, \kappa,(-L)^{-}\right)=0$ :

$$
\hat{a}^{\varepsilon}\left(\omega, \kappa,(-L)^{+}\right)=\varepsilon^{3 / 2}\left(\frac{\hat{f}_{z}(\omega)}{\sqrt{\zeta(\kappa,-L)}}+\frac{i \omega \sqrt{\zeta(\kappa,-L)}}{\rho(-L) i \omega-\varepsilon \sigma(-L)} \kappa \cdot \hat{\boldsymbol{f}}_{\boldsymbol{x}}(\omega)\right) e^{-i \frac{\omega}{\varepsilon} \tau(\kappa,-L)}
$$

We introduce the propagator matrix, that is the matrix such that, for any $-L<$ $z_{0} \leq z \leq 0$ :

$$
\mathbf{P}^{\varepsilon}\left(\omega, \boldsymbol{\kappa}, z_{0}, z\right)\left[\begin{array}{l}
\hat{a}^{\varepsilon}\left(\omega, \boldsymbol{\kappa}, z_{0}\right) \\
\hat{b}^{\varepsilon}\left(\omega, \boldsymbol{\kappa}, z_{0}\right)
\end{array}\right]=\left[\begin{array}{l}
\hat{a}^{\varepsilon}(\omega, \boldsymbol{\kappa}, z) \\
\hat{b}^{\varepsilon}(\omega, \boldsymbol{\kappa}, z)
\end{array}\right]
$$

It is the solution of the linear system

$$
\frac{d \mathbf{P}^{\varepsilon}}{d z}=\mathbf{M}^{\varepsilon}(\omega, \kappa, z) \mathbf{P}^{\varepsilon}
$$

with the initial condition $\mathbf{P}^{\varepsilon}\left(\omega, \boldsymbol{\kappa}, z_{0}, z_{0}\right)=\mathbf{I}$ and the $2 \times 2$ matrix $\mathbf{M}^{\varepsilon}$ given by

$$
\begin{aligned}
& M_{11}^{\varepsilon}(z)=\frac{i \omega c(\kappa, z)}{2 c^{2}(z) \varepsilon} \nu\left(z, \frac{z}{\varepsilon^{2}}\right)-\frac{\sigma(z)}{2 \zeta(z)} \frac{c(\kappa, z)}{c(z)} \\
& M_{21}^{\varepsilon}(z)=\left[\frac{i \omega c(\kappa, z)}{2 c^{2}(z) \varepsilon} \nu\left(z, \frac{z}{\varepsilon^{2}}\right)-\frac{\sigma(z)}{2 \zeta(z)} \frac{c(\kappa, z)}{c(z)}\left(1-2 c^{2}(z) \kappa^{2}\right)+\frac{\partial_{z} \zeta(\kappa, z)}{2 \zeta(\kappa, z)}\right] e^{2 i \frac{\omega}{\varepsilon} \tau(\kappa, z)}, \\
& M_{12}^{\varepsilon}(z)=\left[-\frac{i \omega c(\kappa, z)}{2 c^{2}(z) \varepsilon} \nu\left(z, \frac{z}{\varepsilon^{2}}\right)+\frac{\sigma(z)}{2 \zeta(z)} \frac{c(\kappa, z)}{c(z)}\left(1-2 c^{2}(z) \kappa^{2}\right)+\frac{\partial_{z} \zeta(\kappa, z)}{2 \zeta(\kappa, z)}\right] e^{-2 i \frac{\omega}{\varepsilon} \tau(\kappa, z)}, \\
& M_{22}^{\varepsilon}(z)=-\frac{i \omega c(\kappa, z)}{2 c^{2}(z) \varepsilon} \nu\left(z, \frac{z}{\varepsilon^{2}}\right)+\frac{\sigma(z)}{2 \zeta(z)} \frac{c(\kappa, z)}{c(z)}=-M_{11}^{\varepsilon}(z) .
\end{aligned}
$$

Note that $\mathbf{P}^{\varepsilon}\left(\omega, \boldsymbol{\kappa}, z_{0}, z\right)$ depends on $\boldsymbol{\kappa}$ only through the modulus $\kappa=|\boldsymbol{\kappa}|$. 


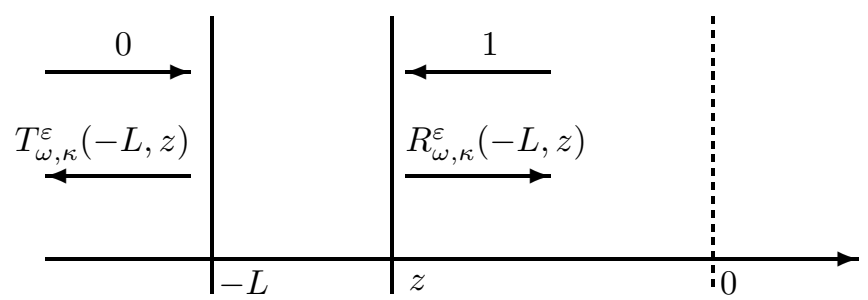

FIG. A.1. Reflection and transmission coefficients.

We introduce the transmission and reflection coefficients $\left(T_{\omega, \kappa}^{\varepsilon}, R_{\omega, \kappa}^{\varepsilon}\right)$ defined by the relation

$$
\mathbf{P}^{\varepsilon}(\omega, \kappa,-L, z)\left[\begin{array}{c}
0 \\
T_{\omega, \kappa}^{\varepsilon}(-L, z)
\end{array}\right]=\left[\begin{array}{c}
R_{\omega, \kappa}^{\varepsilon}(-L, z) \\
1
\end{array}\right],
$$

which corresponds to the scattering problem for a unit-power incident plane wave incoming from the right half-space and probing the inhomogeneous layer in $(-L, z)$ with transparent boundary conditions (see Figure A.1). By inverting this matrixvector relation we get

$$
T_{\omega, \kappa}^{\varepsilon}(-L, z)=\frac{1}{P_{22}^{\varepsilon}(\omega, \kappa,-L, z)}, \quad R_{\omega, \kappa}^{\varepsilon}(-L, z)=\frac{P_{12}^{\varepsilon}(\omega, \kappa,-L, z)}{P_{22}^{\varepsilon}(\omega, \kappa,-L, z)} .
$$

From the relation (A.3) evaluated at $z=0$ and $z_{0}=(-L)^{+}$, we get

$$
\begin{aligned}
\hat{a}^{\varepsilon}(\omega, \kappa, 0) & =\frac{P_{11}^{\varepsilon} P_{22}^{\varepsilon}(\omega, \kappa,-L, 0)-P_{12}^{\varepsilon} P_{21}^{\varepsilon}(\omega, \kappa,-L, 0)}{P_{22}^{\varepsilon}(\omega, \kappa,-L, 0)-P_{12}^{\varepsilon}(\omega, \kappa,-L, 0)} \hat{a}^{\varepsilon}\left(\omega, \kappa,(-L)^{+}\right) \\
& =\frac{\left(\operatorname{det} \mathbf{P}^{\varepsilon}(\omega, \kappa,-L, 0)\right) T_{\omega, \kappa}^{\varepsilon}(-L, 0)}{1-R_{\omega, \kappa}^{\varepsilon}(-L, 0)} \hat{a}^{\varepsilon}\left(\omega, \boldsymbol{\kappa},(-L)^{+}\right) .
\end{aligned}
$$

Since $\mathbf{M}^{\varepsilon}$ has trace zero we have $\partial_{z}\left(\operatorname{det} \mathbf{P}^{\varepsilon}\right)=\left(\operatorname{Tr} \mathbf{M}^{\varepsilon}\right)\left(\operatorname{det} \mathbf{P}^{\varepsilon}\right)=0$, and therefore $\operatorname{det} \mathbf{P}^{\varepsilon}=1$. This simplifies the expression of the transmitted right-going wave amplitude

$$
\hat{a}^{\varepsilon}(\omega, \kappa, 0)=\frac{T_{\omega, \kappa}^{\varepsilon}(-L, 0)}{1-R_{\omega, \kappa}^{\varepsilon}(-L, 0)} \hat{a}^{\varepsilon}\left(\omega, \kappa,(-L)^{+}\right) .
$$

Substituting (A.2) into this expression and taking into account (A.1) gives (3.2) with $T_{\omega, \kappa}^{\varepsilon}=T_{\omega, \kappa}^{\varepsilon}(-L, 0)$ and $R_{\omega, \kappa}^{\varepsilon}=R_{\omega, \kappa}^{\varepsilon}(-L, 0)$.

If we assume that the medium is not dissipative $\sigma \equiv 0$, then $M_{22}^{\varepsilon}=\overline{M_{11}^{\varepsilon}}$ and $M_{12}^{\varepsilon}=\overline{M_{21}^{\varepsilon}}$, which implies that the entries of the propagator matrix satisfy $P_{22}^{\varepsilon}=\overline{P_{11}^{\varepsilon}}$ and $P_{12}^{\varepsilon}=\overline{P_{21}^{\varepsilon}}$. This gives the conservation of energy relation

$$
\begin{aligned}
\left|R_{\omega, \kappa}^{\varepsilon}\right|^{2}+\left|T_{\omega, \kappa}^{\varepsilon}\right|^{2} & =\frac{\left|P_{12}^{\varepsilon}(-L, 0)\right|^{2}+1}{\left|P_{22}^{\varepsilon}(-L, 0)\right|^{2}} \\
& =\frac{P_{12}^{\varepsilon} P_{21}^{\varepsilon}(-L, 0)+P_{11}^{\varepsilon} P_{22}^{\varepsilon}(-L, 0)-P_{12}^{\varepsilon} P_{21}^{\varepsilon}(-L, 0)}{P_{11}^{\varepsilon} P_{22}^{\varepsilon}(-L, 0)} \\
& =1
\end{aligned}
$$

Note that this relation is not valid in a dissipative medium. 
If the medium is homogeneous $c(z)=c_{0}, \zeta(z)=\zeta_{0}, \sigma(z)=\sigma_{0}$ and $\nu \equiv 0$, then the propagator matrix is diagonal

$$
\mathbf{P}^{\varepsilon}(\omega, \kappa,-L, 0)=\left[\begin{array}{cc}
\exp \left(-\frac{\sigma_{0} c_{0}(\kappa)}{2 c_{0}} L\right) & 0 \\
0 & \exp \left(\frac{\sigma_{0} c_{0}(\kappa)}{2 c_{0}} L\right)
\end{array}\right]
$$

and we obtain from (A.2)-(A.7):

$$
\hat{a}^{\varepsilon}(\omega, \boldsymbol{\kappa}, 0)=\varepsilon^{3 / 2}\left(\frac{\hat{f}_{z}(\omega)}{\sqrt{\zeta_{0}(\kappa)}}+\frac{i \omega \sqrt{\zeta_{0}(\kappa)}}{\rho_{0} i \omega-\varepsilon \sigma_{0}} \kappa \cdot \hat{\boldsymbol{f}}_{\boldsymbol{x}}(\omega)\right) \exp \left(\left(\frac{i \omega}{\varepsilon c_{0}(\kappa)}-\frac{\sigma_{0} c_{0}(\kappa)}{2 c_{0}}\right) L\right)
$$

This expression gives (3.8) in Section 3.2.

Appendix B. Proof of Proposition 5.1. By differentiating (A.6) in $z$ and using (A.4) we obtain the closed system satisfied by the reflection and transmission coefficients $R_{\omega, \kappa}^{\varepsilon}(-L, z)$ and $T_{\omega, \kappa}^{\varepsilon}(-L, z)$ :

$$
\begin{aligned}
\frac{d R_{\omega, \kappa}^{\varepsilon}}{d z}= & \frac{i \omega c(\kappa, z)}{2 c^{2}(z) \varepsilon} \nu\left(z, \frac{z}{\varepsilon^{2}}\right)\left(-e^{-2 i \frac{\omega}{\varepsilon} \tau(\kappa, z)}+2 R_{\omega, \kappa}^{\varepsilon}-e^{2 i \frac{\omega}{\varepsilon} \tau(\kappa, z)}\left(R_{\omega, \kappa}^{\varepsilon}\right)^{2}\right) \\
& -\frac{\sigma(z)}{\zeta(z)} \frac{c(\kappa, z)}{c(z)}\left(R_{\omega, \kappa}^{\varepsilon}-\left(1-2 c^{2}(z) \kappa^{2}\right)\left(e^{-2 i \frac{\omega}{\varepsilon} \tau(\kappa, z)}+e^{2 i \frac{\omega}{\varepsilon} \tau(\kappa, z)}\left(R_{\omega, \kappa}^{\varepsilon}\right)^{2}\right)\right. \\
& +\frac{\partial_{z} \zeta(\kappa, z)}{2 \zeta(\kappa, z)}\left(e^{-2 i \frac{\omega}{\varepsilon} \tau(\kappa, z)}-e^{2 i \frac{\omega}{\varepsilon} \tau(\kappa, z)}\left(R_{\omega, \kappa}^{\varepsilon}\right)^{2}\right) \\
\frac{d T_{\omega, \kappa}^{\varepsilon}}{d z}= & \frac{i \omega c(\kappa, z)}{2 c^{2}(z) \varepsilon} \nu\left(z, \frac{z}{\varepsilon^{2}}\right)\left(1-e^{2 i \frac{\omega}{\varepsilon} \tau(\kappa, z)} R_{\omega, \kappa}^{\varepsilon}\right) T_{\omega, \kappa}^{\varepsilon} \\
& -\frac{\sigma(z)}{2 \zeta(z)} \frac{c(\kappa, z)}{c(z)}\left(T_{\omega, \kappa}^{\varepsilon}-\left(1-2 c^{2}(z) \kappa^{2}\right) e^{2 i \frac{\omega}{\varepsilon} \tau(\kappa, z)} R_{\omega, \kappa}^{\varepsilon}\right) \\
& -\frac{\partial_{z} \zeta(\kappa, z)}{2 \zeta(\kappa, z)} e^{2 i \frac{\omega}{\varepsilon} \tau(\kappa, z)} R_{\omega, \kappa}^{\varepsilon} T_{\omega, \kappa}^{\varepsilon},
\end{aligned}
$$

starting from $R_{\omega, \kappa}^{\varepsilon}(-L, z=-L)=0, T_{\omega, \kappa}^{\varepsilon}(-L, z=-L)=1$. This shows that, for a fixed pair $(\omega, \kappa)$ the mode-dependent reflection and transmission coefficients $\left(R_{\omega, \kappa}^{\varepsilon}(-L, z), T_{\omega, \kappa}^{\varepsilon}(-L, z)\right)$ are the solution of the Riccati equations (B.1-B.2). The equations for the mode-dependent reflection and transmission coefficients are uncoupled from the dynamical point of view, because the medium is time-independent and layered. However the equations are statistically coupled because the process $\nu$ appear in all of them, and the correlations between the mode-dependent reflection and transmission coefficients play a crucial role.

The asymptotic behavior of the statistics of the reflection and transmission coefficients can be studied by using diffusion approximation theorems. We only consider the moments of the reflection and transmission coefficients $R_{\omega, \kappa}^{\varepsilon}=R_{\omega, \kappa}^{\varepsilon}(-L, 0)$ and $T_{\omega, \kappa}^{\varepsilon}=T_{\omega, \kappa}^{\varepsilon}(-L, 0)$ that are of interest to our situation. These specific moments of the reflection and transmission coefficients at two nearby slownesses have the following asymptotic behavior as $\varepsilon \rightarrow 0$ [7, Chapters 15 and 17]:

$$
\begin{aligned}
& \mathbb{E}\left[T_{\omega, \kappa+\varepsilon \lambda / 2}^{\varepsilon}\left(R_{\omega, \kappa+\varepsilon \lambda / 2}^{\varepsilon}\right)^{j} \overline{T_{\omega, \kappa-\varepsilon \lambda / 2}^{\varepsilon}\left(R_{\omega, \kappa-\varepsilon \lambda / 2}^{\varepsilon}\right)^{j}}\right] \stackrel{\varepsilon \rightarrow 0}{\longrightarrow} \int \mathcal{V}_{j}(\omega, \kappa, \xi, 0) e^{-i \omega \kappa \lambda \xi} d \xi, \\
& \mathbb{E}\left[T_{\omega, \kappa+\varepsilon \lambda / 2}^{\varepsilon}\left(R_{\omega, \kappa+\varepsilon \lambda / 2}^{\varepsilon}\right)^{j} \overline{T_{\omega, \kappa-\varepsilon \lambda / 2}^{\varepsilon}\left(R_{\omega, \kappa-\varepsilon \lambda / 2}^{\varepsilon}\right)^{l}}\right] \stackrel{\varepsilon \rightarrow 0}{\longrightarrow} 0 \text { if } j \neq l .
\end{aligned}
$$


The $\mathcal{V}_{j}$ 's are the solutions of the system of transport equations

$$
\begin{array}{r}
\frac{\partial \mathcal{V}_{j}}{\partial z}+2 j c(\kappa, z) \frac{\partial \mathcal{V}_{j}}{\partial \xi}=\frac{\gamma(z) c(\kappa, z)^{2} \omega^{2}}{4 c(z)^{4}}\left((j+1)^{2}\left(\mathcal{V}_{j+1}-\mathcal{V}_{j}\right)+j^{2}\left(\mathcal{V}_{j-1}-\mathcal{V}_{j}\right)\right) \\
-\frac{(2 j+1) \sigma(z)}{\zeta(z)} \frac{c(\kappa, z)}{c(z)} \mathcal{V}_{j}
\end{array}
$$

starting from $\mathcal{V}_{j}(\omega, \kappa, \xi, z=-L)=\mathbf{1}_{0}(j) \delta(\xi)$. Here $\gamma(z)$ is the integrated covariance of the fluctuations of the random medium defined by $(2.5)$.

By expanding the generalized transmission coefficient we obtain

$$
\begin{aligned}
\mathbb{E}\left[\mathcal{T}_{\omega, \kappa+\varepsilon \lambda / 2}^{\varepsilon} \overline{\mathcal{T}_{\omega, \kappa-\varepsilon \lambda / 2}^{\varepsilon}}\right] & \stackrel{\varepsilon \rightarrow 0}{\longrightarrow} \int \mathcal{U}(\omega, \kappa, \xi) e^{-i \omega \kappa \lambda \xi} d \xi \\
\mathcal{U}(\omega, \kappa, \xi) & =\sum_{j=0}^{\infty} \mathcal{V}_{j}\left(\omega, \kappa, \xi-\int_{-L}^{0} c(\kappa, z) d z, 0\right)
\end{aligned}
$$

which shows that we need to compute $\sum_{j} \mathcal{V}_{j}(\omega, \kappa, \xi, 0)$.

Eq. (B.5) can be rewritten in terms of the generator $\mathcal{L}_{z}$ defined by (5.3) as:

$$
\frac{\partial \mathcal{V}_{j}}{\partial z}=-2 j c(\kappa, z) \frac{\partial \mathcal{V}_{j}}{\partial \xi}+\left(\mathcal{L}_{z} \mathcal{V}\right)_{j}-\frac{(2 j+1) \sigma(z)}{\zeta(z)} \frac{c(\kappa, z)}{c(z)} \mathcal{V}_{j}
$$

Let us fix some $\xi_{0} \in \mathbb{R}^{+}$and let us introduce the solution $\left(\mathcal{W}_{j}(\omega, \kappa, \xi, z)\right)_{j \in \mathbb{N}}$ of

$$
\frac{\partial \mathcal{W}_{j}}{\partial z}+\left[2 j c(\kappa, z) \frac{\partial \mathcal{W}_{j}}{\partial \xi}+\left(\mathcal{L}_{z} \mathcal{W}\right)_{j}\right]-\frac{(2 j+1) \sigma(z)}{\zeta(z)} \frac{c(\kappa, z)}{c(z)} \mathcal{W}_{j}=0
$$

for $z \in(-L, 0)$, with the terminal condition $\mathcal{W}_{j}(\omega, \kappa, \xi, z=0)=\delta\left(\xi-\xi_{0}\right)$ for all $j \in \mathbb{N}$. Using the fact that the generator $\mathcal{L}_{z}$ is self-adjoint, we have for any $z \in(-L, 0)$ :

$$
\frac{d}{d z}\left[\sum_{j=0}^{\infty} \int \mathcal{V}_{j}(\omega, \kappa, \xi, z) \mathcal{W}_{j}(\omega, \kappa, \xi, z) d \xi\right]=0
$$

and therefore

$$
\sum_{j=0}^{\infty} \int \mathcal{V}_{j} \mathcal{W}_{j}(\omega, \kappa, \xi, z=0) d \xi=\sum_{j=0}^{\infty} \int \mathcal{V}_{j} \mathcal{W}_{j}(\omega, \kappa, \xi, z=-L) d \xi
$$

Taking into account the initial condition satisfied by $\mathcal{V}_{j}$ at $z=-L$ and the terminal condition satisfied by $\mathcal{W}_{j}$ at $z=0$ we find

$$
\sum_{j=0}^{\infty} \mathcal{V}_{j}\left(\omega, \kappa, \xi_{0}, 0\right)=\mathcal{W}_{0}(\omega, \kappa, 0, z=-L)
$$

If we introduce the jump Markov process $\left(N_{\omega, \kappa}(z)\right)_{-L \leq z \leq 0}$ with state space $\mathbb{N}$ and infinitesimal generator $\mathcal{L}_{z}$ and $\left(\xi_{\omega, \kappa}(z)\right)_{-L \leq z \leq 0}$ the process such that

$$
\frac{d \xi_{\omega, \kappa}(z)}{d z}=2 c(\kappa, z) N_{\omega, \kappa}(z),
$$


then the pair $\left(N_{\omega, \kappa}(z), \xi_{\omega, \kappa}(z)\right)_{-L \leq z \leq 0}$ is Markov with generator $2 N c(\kappa, z) \partial_{\xi}+\mathcal{L}_{z}$. Eq. (B.8) can then be interpreted as a Kolmogogov backward equation with a potential. Using Feynmac-Kac formula we find the following probabilistic representation for $\mathcal{W}_{j}$ :

$$
\begin{aligned}
\mathcal{W}_{j}(\omega, \kappa, \xi)= & \mathbb{E}\left[\exp \left(-\int_{-L}^{0} \frac{\sigma(z) c(\kappa, z)}{\zeta(z) c(z)}\left(2 N_{\omega, \kappa}(z)+1\right) d z\right)\right. \\
& \left.\times \delta\left(\xi_{\omega, \kappa}(0)-\xi_{0}\right) \mid \xi_{\omega, \kappa}(-L)=\xi, N_{\omega, \kappa}(-L)=j\right] .
\end{aligned}
$$

Substituting into (B.9) we obtain

$$
\begin{aligned}
\sum_{j=0}^{\infty} \mathcal{V}_{j}\left(\omega, \kappa, \xi_{0}, 0\right)= & \mathbb{E}\left[\delta\left(2 \int_{-L}^{0} c(\kappa, z) N_{\omega, \kappa}(z) d z-\xi_{0}\right)\right. \\
& \left.\times \exp \left(-\int_{-L}^{0} \frac{\sigma(z) c(\kappa, z)}{\zeta(z) c(z)}\left(2 N_{\omega, \kappa}(z)+1\right) d z\right) \mid N_{\omega, \kappa}(-L)=0\right]
\end{aligned}
$$

which gives with (B.7) the probabilistic representation (5.2) for $\mathcal{U}$.

Appendix C. Derivation of Equation (4.2). Let $\phi(\boldsymbol{x})$ and $\psi(t)$ be two smooth test functions compactly supported in the Fourier domain and define the integrated quantity

$$
J^{\varepsilon}=\iint \phi(\boldsymbol{x}) \psi(t) u^{\varepsilon}(t, \boldsymbol{x})^{2} d \boldsymbol{x} d t
$$

Denoting

$$
\hat{g}_{0}^{\varepsilon}(\omega, \boldsymbol{\kappa})=\frac{\hat{f}_{z}(\omega)}{\zeta_{0}(\kappa)}+\frac{i \omega}{i \omega \rho_{0}-\varepsilon \sigma_{0}} \kappa \cdot \hat{\boldsymbol{f}}_{\boldsymbol{x}}(\omega),
$$

we have using (3.2)

$$
\begin{aligned}
J^{\varepsilon}= & \frac{1}{(2 \pi)^{6} \varepsilon^{3}} \iint \hat{g}_{0}^{\varepsilon}\left(\omega_{1}, \boldsymbol{\kappa}_{1}\right) \overline{\hat{g}_{0}^{\varepsilon}}\left(\omega_{2}, \boldsymbol{\kappa}_{2}\right) \mathcal{T}_{\omega_{1},\left|\boldsymbol{\kappa}_{1}\right|}^{\varepsilon} \overline{\mathcal{T}_{\omega_{2},\left|\boldsymbol{\kappa}_{2}\right|}^{\varepsilon}} \\
& \times \overline{\hat{\psi}}\left(\frac{\omega_{1}-\omega_{2}}{\varepsilon}\right) \overline{\hat{\phi}}\left(\frac{\omega_{1} \boldsymbol{\kappa}_{1}-\omega_{2} \boldsymbol{\kappa}_{2}}{\varepsilon}\right) \omega_{1}^{2} \omega_{2}^{2} d \omega_{1} d \omega_{2} d \boldsymbol{\kappa}_{1} d \boldsymbol{\kappa}_{2},
\end{aligned}
$$

where the time and space Fourier transforms are defined by

$$
\hat{\psi}(\omega)=\int \psi(t) e^{i \omega t} d t, \quad \hat{\phi}(\boldsymbol{k})=\int \phi(\boldsymbol{x}) e^{-i \boldsymbol{k} \cdot \boldsymbol{x}} d \boldsymbol{x} .
$$

After the change of variable $\left(\omega_{1}, \omega_{2}, \boldsymbol{\kappa}_{1}, \boldsymbol{\kappa}_{2}\right) \rightarrow(\omega, h, \boldsymbol{\kappa}, \boldsymbol{\lambda})$ such that $\left(\omega_{1}, \omega_{2}, \boldsymbol{\kappa}_{1}, \boldsymbol{\kappa}_{2}\right)=$ $(\omega+\varepsilon h / 2, \omega-\varepsilon h / 2, \boldsymbol{\kappa}+\varepsilon \boldsymbol{\lambda} / 2, \boldsymbol{\kappa}-\varepsilon \boldsymbol{\lambda} / 2)$, we get that, up to smaller terms in $\varepsilon$ :

$$
\begin{aligned}
J^{\varepsilon}=\frac{1}{(2 \pi)^{6}} \iint\left|\hat{g}_{0}^{0}(\omega, \boldsymbol{\kappa})\right|^{2} & \mathcal{T}_{\omega+\varepsilon h / 2,|\boldsymbol{\kappa}+\varepsilon \boldsymbol{\lambda} / 2|} \overline{\mathcal{T}_{\omega-\varepsilon h / 2,|\boldsymbol{\kappa}-\varepsilon \boldsymbol{\lambda} / 2|}^{\varepsilon}} \\
& \times \overline{\hat{\psi}}(h) \overline{\hat{\phi}}(h \boldsymbol{\kappa}+\omega \boldsymbol{\lambda}) \omega^{4} d \omega d h d \boldsymbol{\kappa} d \boldsymbol{\lambda}
\end{aligned}
$$

where $\hat{g}_{0}^{0}(\omega, \boldsymbol{\kappa})=\hat{f}_{z}(\omega) / \zeta_{0}(\kappa)+\boldsymbol{\kappa} \cdot \hat{\boldsymbol{f}}_{\boldsymbol{x}}(\omega) / \rho_{0}$. Note that in (C.2) the integrals in $h$ and $\boldsymbol{\lambda}$ are limited by the test functions (while the integral in $\omega$ is limited by the 
function $\hat{g}_{0}^{0}$ and the integral in $\boldsymbol{\kappa}$ is limited by $|\boldsymbol{\kappa}|<\kappa_{\max }$ given by (3.7)). Moreover, the generalized transmission coefficients have offsets in both frequency and slowness modulus in (C.2). In view of the Riccati equations (B.1-B.2) we see that the offsets affect the rapid phase. Using the expansion

$$
2 \frac{\omega+\varepsilon h / 2}{\varepsilon} \tau(|\boldsymbol{\kappa}+\varepsilon \boldsymbol{\lambda} / 2|, z)=2 \frac{\omega}{\varepsilon} \tau(\kappa, z)+z c_{0}(\kappa)\left[\frac{h}{c_{0}^{2}(\kappa)}-\omega \boldsymbol{\kappa} \cdot \boldsymbol{\lambda}\right]+\mathcal{O}(\varepsilon),
$$

we find via a slight modification of the argument in Appendix B that

$$
\mathbb{E}\left[\mathcal{T}_{\omega+\varepsilon h / 2,|\boldsymbol{\kappa}+\varepsilon \boldsymbol{\lambda} / 2|}^{\varepsilon} \overline{\mathcal{T}_{\omega-\varepsilon h / 2,|\boldsymbol{\kappa}-\varepsilon \boldsymbol{\lambda} / 2|}^{\varepsilon}} \stackrel{\varepsilon \rightarrow 0}{\longrightarrow} \int \mathcal{U}(\omega, \kappa, \xi) e^{i\left[h / c_{0}^{2}(\kappa)-\omega \boldsymbol{\kappa} \cdot \boldsymbol{\lambda}\right] \xi} d \xi\right.
$$

Here $\mathcal{U}$ is defined as in (5.2) in the situation with a constant background medium so that $c(\kappa, z) \equiv c_{0}(\kappa)$. We remark that this expression also follows via replacing the limit in (B.3) by the corresponding limit in [7, Eq. 15.82]. Therefore we obtain

$$
\begin{aligned}
& \lim _{\varepsilon \rightarrow 0} \mathbb{E}\left[J^{\varepsilon}\right]=\frac{1}{(2 \pi)^{3}} \iint\left|\hat{g}_{0}^{0}(\omega, \boldsymbol{\kappa})\right|^{2} \mathcal{U}(\omega, \kappa, \xi) G(\omega, \boldsymbol{\kappa}, \xi) \omega^{2} d \xi d \omega d \boldsymbol{\kappa}, \\
& G(\omega, \boldsymbol{\kappa}, \xi)=\frac{1}{(2 \pi)^{3}} \iint e^{i\left[h / c_{0}^{2}(\kappa)-\omega \boldsymbol{\kappa} \cdot \boldsymbol{\lambda}\right] \xi} \overline{\hat{\psi}}(h) \overline{\hat{\phi}}(h \boldsymbol{\kappa}+\omega \boldsymbol{\lambda}) \omega^{2} d h d \boldsymbol{\lambda} .
\end{aligned}
$$

The function $G$ can be computed:

$$
G(\omega, \boldsymbol{\kappa}, \xi)=\iint \psi(t) \phi(\boldsymbol{x}) \delta(\boldsymbol{x}-\boldsymbol{\kappa} \xi) \delta\left(t-\boldsymbol{\kappa} \cdot \boldsymbol{x}-\frac{\xi}{c_{0}^{2}(\kappa)}\right) d t d \boldsymbol{x}=\psi\left(\frac{\xi}{c_{0}^{2}}\right) \phi(\boldsymbol{\kappa} \xi) .
$$

Substituting into (C.4) and using the change of variables $(\xi, \boldsymbol{\kappa}) \rightarrow(t, \boldsymbol{x})=\left(\xi / c_{0}^{2}, \xi \boldsymbol{\kappa}\right)$ (whose Jacobian is $c_{0}^{2} t^{2}$ ), we find

$$
\begin{aligned}
\lim _{\varepsilon \rightarrow 0} \mathbb{E}\left[J^{\varepsilon}\right] & =\frac{1}{(2 \pi)^{3}} \iint \frac{1}{c_{0}^{2} t^{2}}\left|\hat{g}_{0}^{0}\left(\omega, \frac{\boldsymbol{x}}{c_{0}^{2} t}\right)\right|^{2} \mathcal{U}\left(\omega, \frac{|\boldsymbol{x}|}{c_{0}^{2} t}, c_{0}^{2} t\right) \psi(t) \phi(\boldsymbol{x}) \omega^{2} d \omega d t d \boldsymbol{x} \\
& =\iint I(t, \boldsymbol{x}) \psi(t) \phi(\boldsymbol{x}) d t d \boldsymbol{x}
\end{aligned}
$$

with $I(t, \boldsymbol{x})$ defined by (4.2). This proves the desired result.

Appendix D. Statistical Stability Property. The self-averaging property (4.3) is proved by showing that the variance of the quantity $J^{\varepsilon}$ defined by (C.1) that appears in the left-hand side of (4.3) is going to zero. Using (C.2) the second moment of $J^{\varepsilon}$ is

$$
\begin{aligned}
\mathbb{E}\left[\left(J^{\varepsilon}\right)^{2}\right] & =\frac{1}{(2 \pi)^{12}} \iint\left|\hat{g}_{0}^{0}(\omega, \boldsymbol{\kappa})\right|^{2}\left|\hat{g}_{0}^{0}\left(\omega^{\prime}, \boldsymbol{\kappa}^{\prime}\right)\right|^{2} \\
& \times \mathbb{E}\left[\mathcal{T}_{\omega+\varepsilon h / 2,|\boldsymbol{\kappa}+\varepsilon \boldsymbol{\lambda} / 2|}^{\varepsilon} \overline{\mathcal{T}_{\omega-\varepsilon h / 2,|\boldsymbol{\kappa}-\varepsilon \boldsymbol{\lambda} / 2|}^{\varepsilon}} \mathcal{T}_{\omega^{\prime}+\varepsilon h^{\prime} / 2,\left|\boldsymbol{\kappa}^{\prime}+\varepsilon \boldsymbol{\lambda}^{\prime} / 2\right|}^{\varepsilon} \overline{\mathcal{T}_{\omega^{\prime}-\varepsilon h^{\prime} / 2,\left|\boldsymbol{\kappa}^{\prime}-\varepsilon \boldsymbol{\lambda}^{\prime} / 2\right|}^{\varepsilon}}\right] \\
& \times \overline{\hat{\psi}}(h) \overline{\hat{\phi}}(h \boldsymbol{\kappa}+\omega \boldsymbol{\lambda}) \overline{\hat{\psi}}\left(h^{\prime}\right) \overline{\hat{\phi}}\left(h^{\prime} \boldsymbol{\kappa}^{\prime}+\omega^{\prime} \boldsymbol{\lambda}^{\prime}\right) \omega^{4} \omega^{\prime 4} d \omega d h d \boldsymbol{\kappa} d \boldsymbol{\lambda} d \omega^{\prime} d h^{\prime} d \boldsymbol{\kappa}^{\prime} d \boldsymbol{\lambda}^{\prime},
\end{aligned}
$$

The results obtained in [7, Chapter 14] show that the transmission and reflection coefficients $T_{\omega, \kappa}^{\varepsilon}$ and $R_{\omega, \kappa}^{\varepsilon}$ have a decoherence frequency of the order of $\varepsilon$, and this property is inherited by the generalized transmission coefficient $\mathcal{T}_{\omega, \kappa}^{\varepsilon}$. Using [7, Eq. 14.72] we have, for any $\omega \neq \omega^{\prime}$ :

$$
\begin{aligned}
& \lim _{\varepsilon \rightarrow 0} \mid \mathbb{E}\left[\mathcal{T}_{\omega+\varepsilon h / 2,|\boldsymbol{\kappa}+\varepsilon \boldsymbol{\lambda} / 2|}^{\varepsilon} \overline{\mathcal{T}_{\omega-\varepsilon h / 2,|\boldsymbol{\kappa}-\varepsilon \boldsymbol{\lambda} / 2|}^{\varepsilon}} \mathcal{T}_{\omega^{\prime}+\varepsilon h^{\prime} / 2,\left|\boldsymbol{\kappa}^{\prime}+\varepsilon \boldsymbol{\lambda}^{\prime} / 2\right|}^{\varepsilon} \overline{\mathcal{T}_{\omega^{\prime}-\varepsilon h^{\prime} / 2,\left|\boldsymbol{\kappa}^{\prime}-\varepsilon \boldsymbol{\lambda}^{\prime} / 2\right|}^{\varepsilon}}\right] \\
& -\mathbb{E}\left[\mathcal { T } _ { \omega + \varepsilon h / 2 , | \boldsymbol { \kappa } + \varepsilon \boldsymbol { \lambda } / 2 | } ^ { \varepsilon } \overline { \mathcal { T } _ { \omega - \varepsilon h / 2 , | \boldsymbol { \kappa } - \varepsilon \boldsymbol { \lambda } / 2 | } ^ { \varepsilon } ] } \mathbb { E } \left[\mathcal{T}_{\omega^{\prime}+\varepsilon h^{\prime} / 2,\left|\boldsymbol{\kappa}^{\prime}+\varepsilon \boldsymbol{\lambda}^{\prime} / 2\right|}^{\varepsilon} \overline{\left.\mathcal{T}_{\omega^{\prime}-\varepsilon h^{\prime} / 2,\left|\boldsymbol{\kappa}^{\prime}-\varepsilon \boldsymbol{\lambda}^{\prime} / 2\right|}^{\varepsilon}\right]} \mid=0\right.\right.
\end{aligned}
$$


and we also have (from the next section) that the fourth-order moments of the generalized transmission coefficient are uniformly bounded in $\varepsilon$ for $\omega, \omega^{\prime}, h, h^{\prime}$ in compact domains. Therefore we obtain

$$
\begin{array}{rl}
\lim _{\varepsilon \rightarrow 0} & \mathbb{E}\left[\left(J^{\varepsilon}\right)^{2}\right]=\frac{1}{(2 \pi)^{12}} \iint\left|\hat{g}_{0}^{0}(\omega, \boldsymbol{\kappa})\right|^{2}\left|\hat{g}_{0}^{0}\left(\omega^{\prime}, \boldsymbol{\kappa}^{\prime}\right)\right|^{2} \\
\quad \times \lim _{\varepsilon \rightarrow 0} \mathbb{E}\left[\mathcal{T}_{\omega+\varepsilon h / 2,|\boldsymbol{\kappa}+\varepsilon \boldsymbol{\lambda} / 2|}^{\varepsilon} \overline{\mathcal{T}_{\omega-\varepsilon h / 2,|\boldsymbol{\kappa}-\varepsilon \boldsymbol{\lambda} / 2|}^{\varepsilon}} \mathbb{E}\left[\mathcal{T}_{\omega^{\prime}+\varepsilon h^{\prime} / 2,\left|\boldsymbol{\kappa}^{\prime}+\varepsilon \boldsymbol{\lambda}^{\prime} / 2\right|}^{\varepsilon} \overline{\mathcal{T}_{\omega^{\prime}-\varepsilon h^{\prime} / 2,\left|\boldsymbol{\kappa}^{\prime}-\varepsilon \boldsymbol{\lambda}^{\prime} / 2\right|}^{\varepsilon}}\right]\right. \\
\times \overline{\hat{\psi}}(h) \overline{\hat{\phi}}(h \boldsymbol{\kappa}+\omega \boldsymbol{\lambda}) \overline{\hat{\psi}}\left(h^{\prime}\right) \overline{\hat{\phi}}\left(h^{\prime} \boldsymbol{\kappa}^{\prime}+\omega^{\prime} \boldsymbol{\lambda}^{\prime}\right) \omega^{4} \omega^{\prime 4} d \omega d h d \boldsymbol{\kappa} d \boldsymbol{\lambda} d \omega^{\prime} d h^{\prime} d \boldsymbol{\kappa}^{\prime} d \boldsymbol{\lambda}^{\prime} \\
\quad=\lim _{\varepsilon \rightarrow 0} \mathbb{E}\left[J^{\varepsilon}\right]^{2},
\end{array}
$$

which shows that the variance of $J^{\varepsilon}$ goes to zero as $\varepsilon \rightarrow 0$.

Appendix E. Higher-order Moments of the Generalized Transmission Coefficient. The goal of this appendix is to compute the limiting moments of the generalized transmission coefficient of the form

$$
\lim _{\varepsilon \rightarrow 0} \mathbb{E}\left[\left(\mathcal{T}_{\omega, \kappa+\varepsilon \lambda / 2}^{\varepsilon}\right)^{k}\left(\overline{\mathcal{T}_{\omega, \kappa-\varepsilon \lambda / 2}^{\varepsilon}}\right)^{k}\right],
$$

for any integer $k \geq 2$. The method follows the lines of the one used in Appendix B. We first consider the limits of the following moments of reflection and transmission coefficients:

$$
\begin{array}{r}
\mathbb{E}\left[\left(T_{\omega, \kappa+\varepsilon \lambda / 2}^{\varepsilon}\right)^{k}\left(R_{\omega, \kappa+\varepsilon \lambda / 2}^{\varepsilon}\right)^{j} \overline{\left(T_{\omega, \kappa-\varepsilon \lambda / 2}^{\varepsilon}\right)^{k}\left(R_{\omega, \kappa-\varepsilon \lambda / 2}^{\varepsilon}\right)^{j}}\right] \\
\stackrel{\varepsilon \rightarrow 0}{\longrightarrow} \int \mathcal{V}_{j}^{(k)}(\omega, \kappa, \xi, 0) e^{-i \omega \kappa \lambda \xi} d \xi
\end{array}
$$

where the $\mathcal{V}_{j}^{(k)}$ 's are the solutions of the systems of transport equations

$$
\begin{array}{r}
\frac{\partial \mathcal{V}_{j}^{(k)}}{\partial z}+2 j c(\kappa, z) \frac{\partial \mathcal{V}_{j}^{(k)}}{\partial \xi}=\frac{\gamma(z) c(\kappa, z)^{2} \omega^{2}}{4 c(z)^{4}}\left((j+k)^{2}\left(\mathcal{V}_{j+1}^{(k)}-\mathcal{V}_{j}^{(k)}\right)+j^{2}\left(\mathcal{V}_{j-1}^{(k)}-\mathcal{V}_{j}^{(k)}\right)\right) \\
-\frac{(2 j+k) \sigma(z)}{\zeta(z)} \frac{c(\kappa, z)}{c(z)} \mathcal{V}_{j}^{(k)}
\end{array}
$$

starting from $\mathcal{V}_{j}^{(k)}(\omega, \kappa, \xi, z=-L)=\mathbf{1}_{0}(j) \delta(\xi)$. By expanding the $k$-th power of the generalized transmission coefficient $(3.6)$ :

$$
\begin{aligned}
& \left(\mathcal{T}_{\omega, \kappa}^{\varepsilon}\right)^{k}=\exp \left(-i k \frac{\omega}{\varepsilon} \tau(\kappa,-L)\right) \sum_{j=0}^{\infty} \beta^{(k)}(j)\left(T_{\omega, \kappa}^{\varepsilon}\right)^{k}\left(R_{\omega, \kappa}^{\varepsilon}\right)^{j} \\
& \beta^{(k)}(j)=\frac{(j+k-1) !}{j !(k-1) !}
\end{aligned}
$$

we obtain

$$
\begin{aligned}
\mathbb{E}\left[\left(\mathcal{T}_{\omega, \kappa+\varepsilon \lambda / 2}^{\varepsilon}\right)^{k}\left(\frac{\mathcal{T}_{\omega, \kappa-\varepsilon \lambda / 2}^{\varepsilon}}{k}\right]\right. & \stackrel{\varepsilon \rightarrow 0}{\longrightarrow} \int \mathcal{U}^{(k)}(\omega, \kappa, \xi) e^{-i \omega \kappa \lambda \xi} d \xi \\
\mathcal{U}^{(k)}(\omega, \kappa, \xi) & =\sum_{j=0}^{\infty} \beta^{(k)}(j)^{2} \mathcal{V}_{j}^{(k)}\left(\omega, \kappa, \xi-k \int_{-L}^{0} c(\kappa, z) d z, 0\right) .
\end{aligned}
$$


We then find the following probabilistic representation for $\mathcal{U}^{(k)}(\omega, \kappa, \xi)$ :

$$
\begin{aligned}
\mathcal{U}^{(k)}(\omega, \kappa, \xi)= & \mathbb{E}\left[\beta^{(k)}\left(N_{\omega, \kappa}^{(k)}(0)\right)^{2} \delta\left(\xi-\int_{-L}^{0} c(\kappa, z)\left(2 N_{\omega, \kappa}^{(k)}(z)+k\right) d z\right)\right. \\
& \left.\times \exp \left(-\int_{-L}^{0} \frac{\sigma(z) c(\kappa, z)}{\zeta(z) c(z)}\left(2 N_{\omega, \kappa}^{(k)}(z)+k\right) d z\right) \mid N_{\omega, \kappa}^{(k)}(-L)=0\right]
\end{aligned}
$$

where $\left(N_{\omega, \kappa}^{(k)}(z)\right)_{-L \leq z \leq 0}$ is the jump Markov process with state space $\mathbb{N}$ and inhomogeneous infinitesimal generator

$$
\mathcal{L}_{z}^{(k)} \phi(N)=\frac{\gamma(z) c(\kappa, z)^{2} \omega^{2}}{4 c(z)^{4}}\left[(N+k)^{2}(\phi(N+1)-\phi(N))+N^{2}(\phi(N-1)-\phi(N))\right] .
$$

In particular, in absence of dissipation $\sigma(z) \equiv 0$, we obtain $\left(\right.$ since $\left.\beta^{(2)}(j)=j+1\right)$ :

$$
\lim _{\varepsilon \rightarrow 0} \mathbb{E}\left[\left|\mathcal{T}_{\omega, \kappa}^{\varepsilon}\right|^{4}\right]=\int \mathcal{U}^{(2)}(\omega, \kappa, \xi) d \xi=\mathbb{E}\left[\left(N_{\omega, \kappa}^{(2)}(0)+1\right)^{2} \mid N_{\omega, \kappa}^{(2)}(-L)=0\right]
$$

Using the fact that, for $\phi(N)=(N+1)^{2}$, we have

$$
\mathcal{L}_{z}^{(2)} \phi(N)=\frac{\gamma(z) c(\kappa, z)^{2} \omega^{2}}{2 c(z)^{4}}[5 \phi(N)+1]
$$

we can solve the Kolmogorov backward equation and find that

$$
\lim _{\varepsilon \rightarrow 0} \mathbb{E}\left[\left|\mathcal{T}_{\omega, \kappa}^{\varepsilon}\right|^{4}\right]=\frac{6}{5} \exp \left(\int_{-L}^{0} \frac{5 \gamma(z) c(\kappa, z)^{2} \omega^{2}}{2 c(z)^{4}} d z\right)-\frac{1}{5}
$$

which gives the desired result (7.2).

Appendix F. Proof of Proposition 7.5. By using (4.2) and Lemma 5.1, we find that, for any $q \geq 0$ :

$$
\int t^{2+q} I(t, \mathbf{0}) d t=\frac{1}{(2 \pi)^{3} c_{0}^{4+q} \zeta_{0}^{2}} \int \mathbb{E}_{0}\left[\left(\int_{-L}^{0} 2 N_{\omega, 0}(z)+1 d z\right)^{q}\right]\left|\hat{f}_{z}(\omega)\right|^{2} \omega^{2} d \omega
$$

where $\left(N_{\omega, 0}(z)\right)_{-L \leq z \leq 0}$ is the jump process with the homogeneous infinitesimal generator $\mathcal{L}_{\omega, 0}$ and $\mathbb{E}_{0}[\cdot]$ is the expectation with respect to the jump process $N_{\omega, 0}$ starting from $N_{\omega, 0}(-L)=0$. The moments of $N_{\omega, \kappa}$ up to order $q$ satisfy a closed linear system that can be solved. In particular we have

$$
\frac{d \mathbb{E}_{0}\left[N_{\omega, 0}(z)\right]}{d z}=\mathbb{E}_{0}\left[\mathcal{L}_{\omega, 0} N_{\omega, 0}(z)\right]=\frac{\gamma_{0} \omega^{2}}{4 c_{0}^{2}}\left(2 \mathbb{E}_{0}\left[N_{\omega, 0}(z)\right]+1\right),
$$

so that we obtain

$$
\mathbb{E}_{0}\left[N_{\omega, 0}(z)\right]=\frac{1}{2}\left[\exp \left(\frac{\gamma_{0} \omega^{2}}{2 c_{0}^{2}}(L+z)\right)-1\right]
$$

which gives:

$$
\int t^{3} I(t, \mathbf{0}) d t=\frac{1}{(2 \pi)^{3} c_{0}^{5} \zeta_{0}^{2}} \int \frac{2 c_{0}^{2}}{\gamma_{0} \omega^{2}}\left[\exp \left(\frac{\gamma_{0} \omega^{2}}{2 c_{0}^{2}} L\right)-1\right]\left|\hat{f}_{z}(\omega)\right|^{2} \omega^{2} d \omega
$$


and the proposition now follows.

Appendix G. Proof of Proposition 7.6. Taking into account the fact that the jump process can jump only between $-z_{0}$ and $-z_{0}+\Delta z$, the probabilistic representation of the spectral density can be written in the form

$$
\begin{aligned}
\mathcal{U}(\omega, \kappa, \xi)= & \mathbb{E}\left[\delta \left(\xi-c_{0}(\kappa) L-2 c_{0}(\kappa) \int_{-z_{0}}^{-z_{0}+\Delta z} N_{\omega, \kappa}(z) d z\right.\right. \\
& \left.\left.-2 c_{0}(\kappa)\left(z_{0}-\Delta z\right) N_{\omega, \kappa}\left(-z_{0}+\Delta z\right)\right) \mid N_{\omega, \kappa}\left(-z_{0}\right)=0\right]
\end{aligned}
$$

where $\left(N_{\omega, \kappa}(z)\right)_{-L \leq z \leq 0}$ is the jump process with the infinitesimal generator $\mathcal{L}_{\omega, \kappa}$ defined by (6.4). In the limit case of a thin and strong scattering layer $\Delta z \rightarrow 0$ and $\gamma_{0} \Delta z \rightarrow \widetilde{\gamma_{0}}>0$, we thus find

$$
\begin{aligned}
\mathcal{U}(\omega, \kappa, \xi) & =\mathbb{E}\left[\delta\left(\xi-c_{0}(\kappa) L-2 c_{0}(\kappa) z_{0} \widetilde{N}_{\omega, \kappa}(1)\right) \mid \widetilde{N}_{\omega, \kappa}(0)=0\right] \\
& =\sum_{n=0}^{\infty} \mathbb{P}\left(\widetilde{N}_{\omega, \kappa}(1)=n \mid \widetilde{N}_{\omega, \kappa}(0)=0\right) \delta\left(\xi-c_{0}(\kappa) L-2 n c_{0}(\kappa) z_{0}\right)
\end{aligned}
$$

where $\widetilde{N}_{\omega, \kappa}$ is the jump Markov process with the homogeneous infinitesimal generator:

$$
\widetilde{\mathcal{L}}_{\omega, \kappa} \phi(N)=\Gamma_{\omega, \kappa}\left[(N+1)^{2}(\phi(N+1)-\phi(N))+N^{2}(\phi(N-1)-\phi(N))\right] .
$$

The intensity at the surface at $(\boldsymbol{x}, 0)$ is therefore a series of the form:

$$
\begin{aligned}
& I(t, \boldsymbol{x})=\frac{S_{n} O^{2}}{(2 \pi)^{3} \zeta_{0}^{2} c_{0}^{2} S_{n} M^{4}} \\
& \times \sum_{n=0}^{\infty} \mathbb{P}\left(\tilde{N}_{\omega, \kappa_{n}}(1)=n \mid \tilde{N}_{\omega, \kappa}(0)=0\right) \delta\left(t-\frac{S_{n} M}{c_{0}}\right) \int\left|\frac{\overrightarrow{S_{n} M}}{S_{n} M} \cdot \hat{\overrightarrow{\boldsymbol{f}}}(\omega)\right|^{2} \omega^{2} d \omega .
\end{aligned}
$$

Using (B.3), (B.5), and the probabilistic representation given in Proposition 5.1 we find

$$
\mathbb{P}\left(\widetilde{N}_{\omega, \kappa}(1)=0 \mid \widetilde{N}_{\omega, \kappa}(0)=n\right)=\lim _{\varepsilon \rightarrow 0} \mathbb{E}\left[\left|\widetilde{T}_{\omega, \kappa}^{\varepsilon}\right|^{2}\left|\widetilde{R}_{\omega, \kappa}^{\varepsilon}\right|^{2 n}\right]
$$

with $\widetilde{T}_{\omega, \kappa}^{\varepsilon}, \widetilde{R}_{\omega, \kappa}^{\varepsilon}$ being the transmission and reflection coefficients associated with the section $(0,1)$ for the medium corresponding to the generator $\widetilde{\mathcal{L}}_{\omega, \kappa}$. Moreover, using the self-adjointness of $\widetilde{\mathcal{L}}_{\omega, \kappa}$ we have $\mathbb{P}\left(\widetilde{N}_{\omega, \kappa}(1)=0 \mid \widetilde{N}_{\omega, \kappa}(0)=n\right)=\mathbb{P}\left(\widetilde{N}_{\omega, \kappa}(1)=n \mid\right.$ $\left.\widetilde{N}_{\omega, \kappa}(0)=0\right)$, which then shows that the amplitudes of the multiples are decaying in $n$ since $\left|\widetilde{R}_{\omega, \kappa}^{\varepsilon}\right| \leq 1$. Using the energy conservation relation (A.8), we get

$$
\mathbb{P}\left(\tilde{N}_{\omega, \kappa}=n \mid \tilde{N}_{\omega, \kappa}(0)=0\right)=\sum_{j=0}^{n}\left(\begin{array}{c}
n \\
j
\end{array}\right)(-1)^{j} \lim _{\varepsilon \rightarrow 0} \mathbb{E}\left[\left|\widetilde{T}_{\omega, \kappa}^{\varepsilon}\right|^{2 j+2}\right]
$$

The limits of the high-order moments of the power transmission coefficient $\left|\widetilde{T}_{\omega, \kappa}^{\varepsilon}\right|^{2}$ are given in [7, Section 7.1.5]:

$$
\lim _{\varepsilon \rightarrow 0} \mathbb{E}\left[\left|\widetilde{T}_{\omega, \kappa}^{\varepsilon}\right|^{2 j+2}\right]=\Xi_{j+1}\left(\Gamma_{\omega, \kappa}\right)
$$

with the notations introduced in Proposition 7.6. This gives the expression of the amplitudes of the multiples. The proposition now follows with $\alpha_{n}(\omega, \kappa)=\mathbb{P}\left(\widetilde{N}_{\omega, \kappa}(1)=\right.$ $\left.n \mid \widetilde{N}_{\omega, \kappa}(0)=0\right)$. 


\section{REFERENCES}

[1] M. Asch, W. Kohler, G. Papanicolaou, M. Postel, and B. White, Frequency content of randomly scattered signals, SIAM Review, 33 (1991), pp. 519-626.

[2] L. Borcea, F. Gonzalez del Cueto, G. Papanicolaou, and C. Tsogka, Filtering deterministic layering effects in imaging, SIAM Multiscale Model. Simul., 7 (2009), pp. 1267-1301.

[3] L. Borcea, G. Papanicolaou, and C. Tsogka, Coherent interferometry in finely layered random media, SIAM Multiscale Model. Simul., 5 (2006), pp. 62-83.

[4] J. Chillan and J.-P. Fouque, Pressure fields generated by acoustical pulses propagating in randomly layered media, SIAM J. Appl. Math., 58 (1998), pp. 1532-1546.

[5] A. T. de Hoop, Handbook of radiation and scattering of waves, Academic Press, London, 1995. Available via http://www.atdehoop.com.

[6] J.-P. Fouque, J. Garnier, A. Nachbin, and K. Sølna, Time reversal refocusing for point source in randomly layered media, Wave Motion, 42 (2005), pp. 238-260.

[7] J.-P. Fouque, J. Garnier, G. Papanicolaou, and K. Sølna, Wave propagation and time reversal in randomly layered media, Springer, New York, 2007.

[8] J.-P. Fouque, J. Garnier, and K. Sølna, Time reversal super resolution in randomly layered media, Wave Motion, 43 (2006), pp. 646-666.

[9] J. Garnier, Imaging in randomly layered media by cross-correlating noisy signals, SIAM Multiscale Model. Simul., 4 (2005), pp. 610-640.

[10] J. Garnier and K. Sølna, Coupled paraxial wave equations in random media in the white-noise regime, Ann. Appl. Probab., 19 (2009), pp. 318-346.

[11] S. A. Gredeskul and V. D. Freilikher, Waveguiding properties of randomly stratified media, Radiofiz., 31 (1988), pp. 1210-1217 (pp. 861-867 in English).

[12] J. Keller, G. Papanicolaou, and L. Ryzhik, Transport equations for elastic and other waves in random media, Wave Motion, 24 (1996), pp. 327-370.

[13] W. Kohler, G. Papanicolaou, and B. White, Reflection of waves generated by a point source over a randomly layered medium, Wave Motion, 13 (1991), pp. 53-87.

[14] W. Kohler, G. Papanicolaou, and B. White, Reflection and transmission of acoustic waves by a locally-layered slab, in: Diffuse Waves in Complex Media (Les Houches, 1998), J.-P. Fouque, ed., NATO Sci. Ser. C Math. Phys. Sci., Vol. 531, Kluwer Acad. Publ., Dordrecht, 1999, pp. 347-382.

[15] B. M. Shevtsov, Statistical characteristics for wave packet scattering in a layered randomly inhomogeneous medium above a reflecting surface, Radiofiz., 30 (1987), pp. 1007-1012 (pp. 750-754 in English).

[16] K. Sølna and G. Papanicolaou, Ray theory for a locally layered medium, Waves Random Media, 10 (2000), pp. 155-202.

[17] B. Ursin, Review of elastic and electromagnetic wave propagation in horizontally layered media, Geophysics, 48 (1983), pp. 1063-1081.

[18] B. White, P. Sheng, and B. Nair, Localization and backscattering spectrum of seismic waves in stratified lithology, Geophysics, 55 (1990), pp. 1158-1165. 